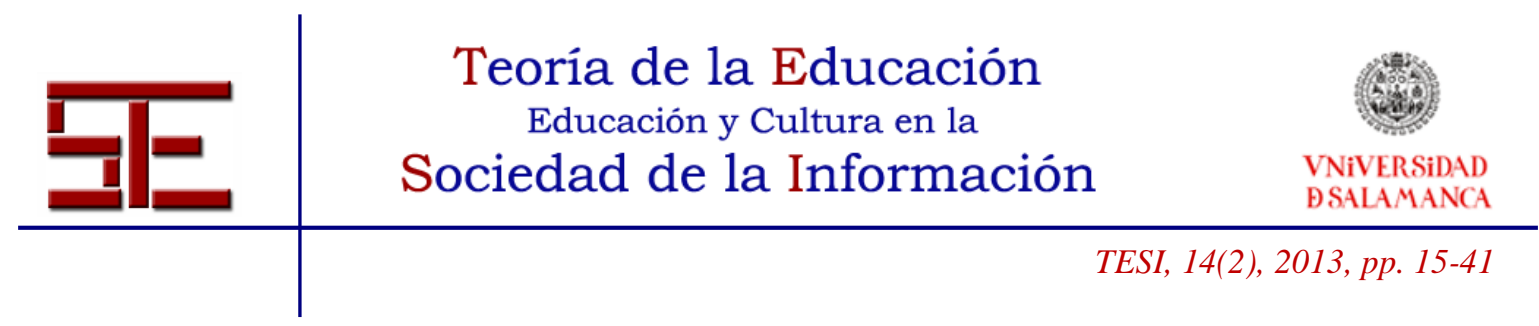

\title{
APRENDIZAJE ESTRATÉGICO Y TECNOLOGÍAS DE LA INFORMACIÓN Y LA COMUNICACIÓN: UNA REVISIÓN CRÍTICA
}

Resumen: En este artículo se presenta un esquema de redefinición de estrategia de aprendizaje, basada en las contribuciones recientes de las tecnologías del aprendizaje. Esta nueva definición aborda la cuestión de cómo las posibilidades tecnológicas de los programas informáticos, de las herramientas tecnológicas, o de los entornos educativos, hacen posible que un alumno o un grupo de alumnos utilicen nuevas formas de desarrollar un aprendizaje estratégico. Revisamos cómo diferentes características de la definición de estrategia de aprendizaje se han transformado debido a la introducción de las TIC en la educación. Por último, el artículo señala nuevos desafíos conceptuales y futuros desarrollos de la investigación.

Palabras clave: Estrategias de aprendizaje; usos educativos de la tecnología; competencia informacional.

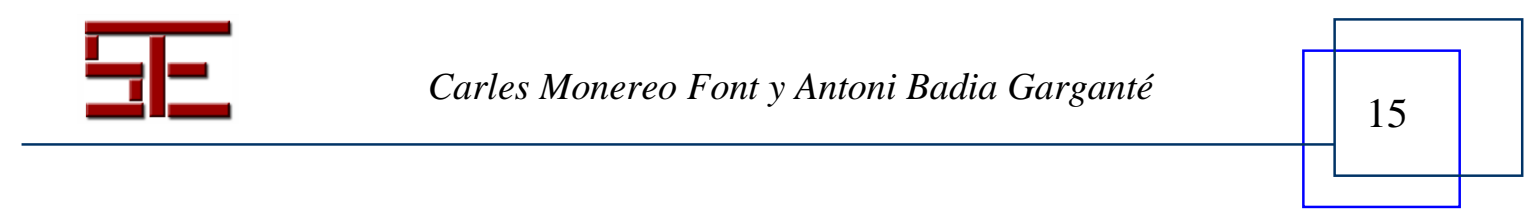




\title{
STRATEGIC LEARNING AND INFORMATION AND COMMUNICATION TECHNOLOGY: A CRITICAL REVIEW
}

\begin{abstract}
This article presents an outline of a learning strategy redefinition, based on recent contributions from learning technologies. This new definition addresses the question of how technological affordances of the software, of the technological tools, or an educational environment, allow a learner or a group of learners develop a new ways to perform a strategic learning action. We review how different characteristics of learning strategy definition have been transformed because of introduction of ICT into education. Finally, the article points toward conceptual challenges and future research questions.
\end{abstract}

Keywords: learning strategies; educational uses of technology; informational competency.

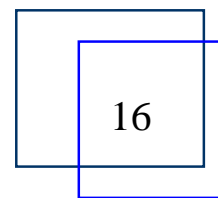




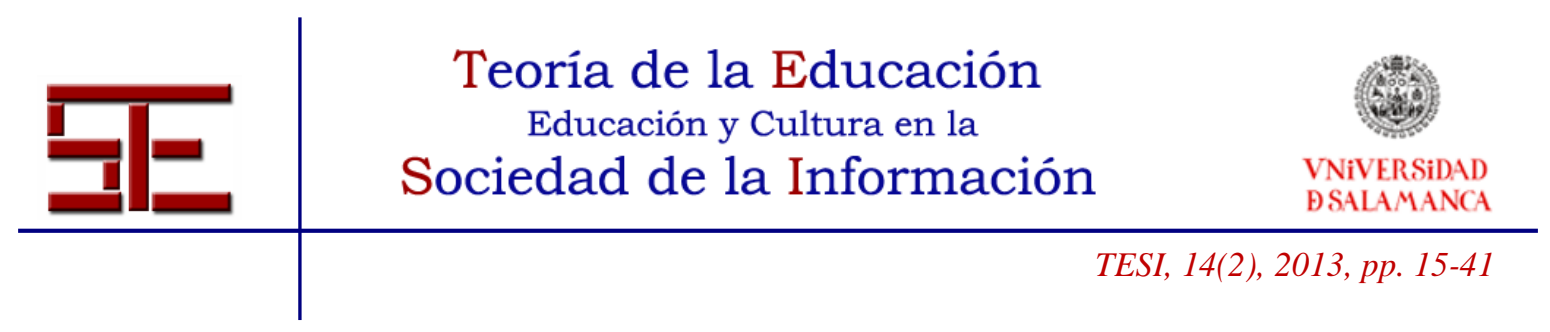

\section{APRENDIZAJE ESTRATÉGICO Y TECNOLOGÍAS DE LA INFORMACIÓN Y LA COMUNICACIÓN: UNA REVISIÓN CRÍTICA}

Fecha de recepción: 05/03/2013; fecha de aceptación: 26/06/2013; fecha de publicación: 30/07/2013

Carles Monereo Font

carles.monereo@uab.cat

Universidad Autónoma de Barcelona

Antoni Badia Garganté

tbadia@uoc.edu

Universidad Oberta de Catalunya

\section{1.- INTRODUCCIÓN}

Este artículo tiene como principal finalidad proponer una re-conceptualización de la noción de estrategia de aprendizaje (a partir de aquí, EA) que tenga en cuenta el impacto que está teniendo la integración de las Tecnologías de la información y la Comunicación (a partir de aquí, TIC) en la educación. En particular, nos centraremos en caracterizar de qué modo los posibles usos educativos de las TIC desplegados en las aulas pueden modificar las estrategias que los alumnos utilizan para aprender los contenidos curriculares.

Para lograr esta finalidad hemos estructurado el artículo en cuatro partes interrelacionadas. Consideramos que, en primer lugar, es necesario realizar un breve recorrido histórico para constatar de qué modo los usos educativos de las TIC han influido sobre la evolución de las estrategias de aprendizaje, a lo largo de los últimos años. Ello nos será útil para conocer mejor por qué se realizan determinadas propuestas teóricas en la actualidad. Partiendo de la última definición mostrada de las nociones de usos educativos de las TIC y de estrategia de aprendizaje, en segundo lugar nos proponemos revisar los cambios conceptuales que deberían hacerse para que dicha noción de estrategia de aprendizaje asuma los cambios teóricos necesarios que provienen del uso de las TIC en los procesos de aprendizaje de los alumnos. Seguidamente, ejemplificaremos un proceso de aprendizaje estratégico de un contenido curricular específico realizado mediante el uso intensivo de las TIC. Para terminar, en cuarto lugar reflexionaremos sobre algunos retos en la investigación futura sobre la nueva conceptualización de estrategias de aprendizaje que se propone en este artículo.

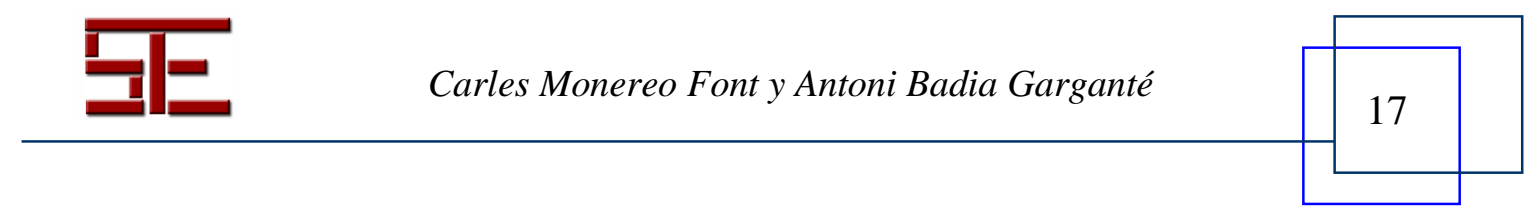




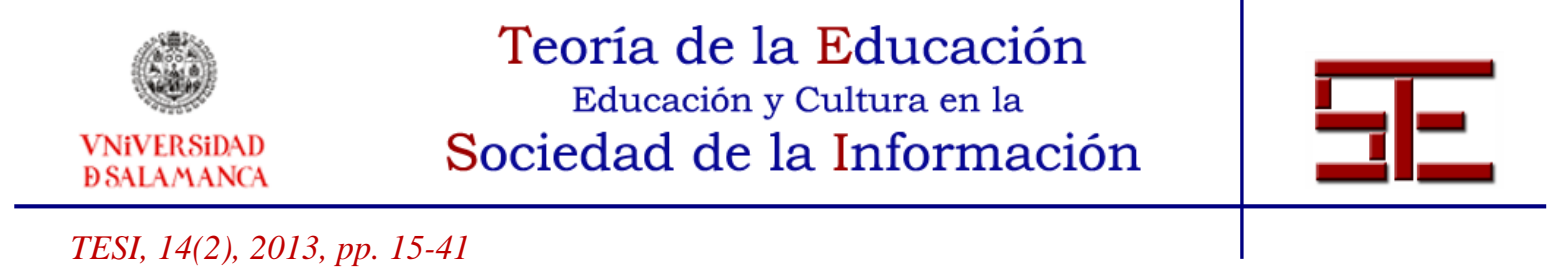

\section{2.- ESTRATEGIAS DE APRENDIZAJE Y LOS USOS EDUCATIVOS DE LAS TIC}

Siguiendo la propuesta de varios autores (Badia, Álvarez, Carretero, Liesa y Becerril, 2012; Badia y Monereo, 2008; Gargallo, 2012) consideramos que, en las tres últimas décadas, los usos educativos de las TIC pueden ser conceptualizados, como mínimo, de tres formas diferentes. Cronológicamente, los tres tipos de conceptualizaciones que presentamos a continuación tienen su correlato con tres formas particulares de concebir las estrategias de aprendizaje en el marco de una tarea de aprendizaje.

\begin{tabular}{|c|l|l|}
\hline & Concepciones de los usos educativos de las TIC & Concepciones de las EA \\
\hline 1 & $\begin{array}{l}\text { Las TIC como herramientas diseñadas para un uso } \\
\text { generalizado en los procesos de enseñanza-aprendizaje }\end{array}$ & $\begin{array}{l}\text { Como procesos } \\
\text { cognitivos generales }\end{array}$ \\
\hline 2 & $\begin{array}{l}\text { Las TIC como sistemas tutoriales diseñados para guiar } \\
\text { los procesos cognitivos y aprender determinados } \\
\text { procedimientos vinculados con tareas educativas } \\
\text { prototípicas }\end{array}$ & $\begin{array}{l}\text { Como procesos } \\
\text { cognitivos específicos }\end{array}$ \\
\hline 3 & $\begin{array}{l}\text { Las TIC como instrumentos relacionados con la toma } \\
\text { de decisiones para resolver tareas educativas abiertas o o } \\
\text { problemáticas (p. e.: guiones -scripts- integrados en } \\
\text { tareas auténticas). }\end{array}$ & $\begin{array}{l}\text { Como procesos } \\
\text { cognitivos socialmente } \\
\text { situados de toma de } \\
\text { decisiones }\end{array}$ \\
\hline
\end{tabular}

Tabla 1. Interrelación entre las concepciones de los usos educativos de las TIC y las concepciones de estrategias de aprendizaje

El primer tipo de aproximación conceptual supone hacer corresponder los usos educativos de las TIC con los usos de los diversos programas informáticos existentes, disponibles de forma generalizada, aplicados a la educación con finalidades de enseñanza y aprendizaje. En el marco de este tipo de usos de las TIC, las estrategias de aprendizaje son consideradas procesos cognitivos generales desplegados en el marco de tareas de aprendizaje. Se trata de clasificaciones de habilidades generales (tales como acceder, seleccionar, memorizar, elaborar, clasificar o comunicar información, entre otros) que pueden ser aplicadas a cualquier tipo de contenido curricular. Los estudios típicos realizados desde esta perspectiva proponen clasificaciones de las TIC a dos niveles. En un nivel más básico (ver, por ejemplo, Selwyn y Bullon, 2000) se centran en identificar el uso de las TIC por parte de los alumnos para dibujar, para escribir, para realizar cálculos matemáticos o para acceder a información de Internet, entre otros. En otras clasificaciones un poco más sofisticadas (ver, por ejemplo Lim y Tay, 2003), se realizan propuestas que

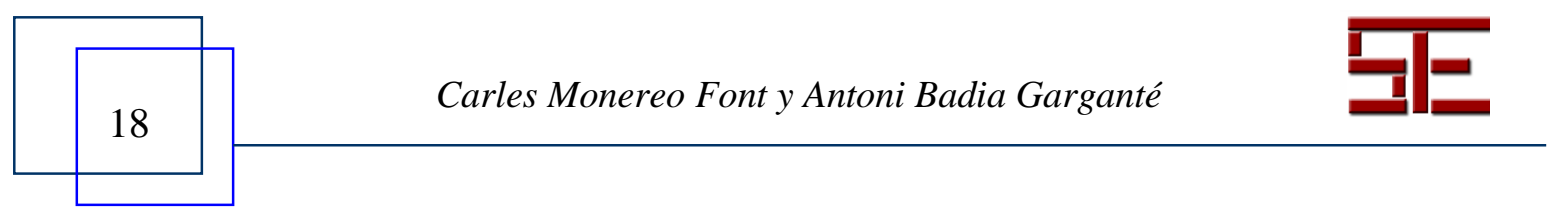




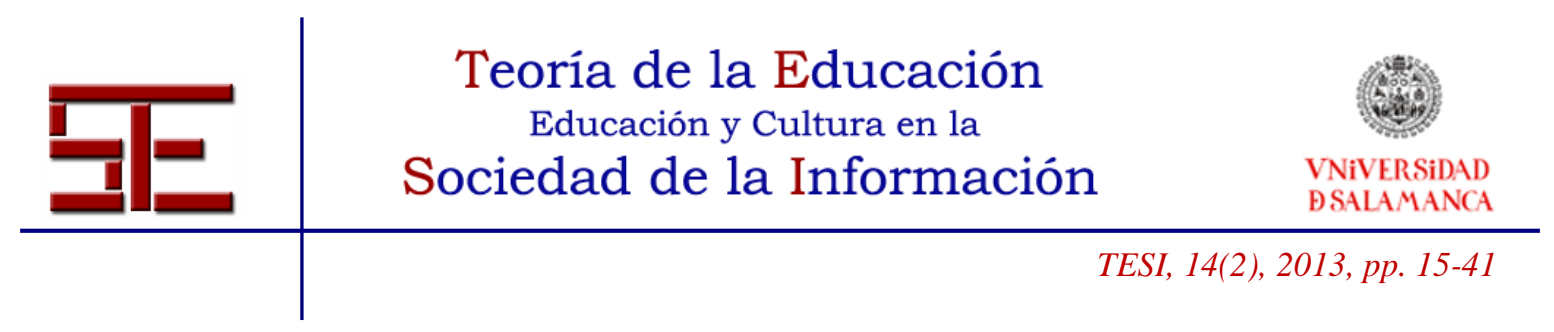

involucran procesos de pensamiento de orden superior, vinculados con el uso de las TIC como herramientas para acceder, elaborar, estructurar y comunicar información sobre los contenidos curriculares.

El segundo tipo de enfoque conceptual supone concebir las TIC como "instrumentos cognitivos" (Jonassen, 2006; Lajoie y Derry, 1993), entendidos como herramientas que son usadas para dar soporte a los procesos cognitivos de los alumnos en su aprendizaje. Desde esta perspectiva, las estrategias se consideran procesos cognitivos situados en contextos educativos concretos, aplicados a tareas prototípicas de aprendizaje en áreas curriculares específicas. Desde esta formulación, las TIC pueden ser usadas como sistemas tutoriales diseñados para guiar y orientar los procesos cognitivos que los alumnos despliegan en tareas específicas de aprendizaje, como por ejemplo la resolución de problemas matemáticos, la lectura de textos, o la escritura argumentativa. Un caso típico de esta interrelación entre estrategias de aprendizaje y usos de las TIC son, por ejemplo, los sistemas tutoriales aplicados a los procesos de resolución de problemas (Reusser, 1993). En este caso particular, el sistema tutorial fue diseñado para guiar al alumno en los procesos de representación de información relacionada con la comprensión del problema, la planificación de la ejecución y la reflexión del proceso seguido en la resolución de problemas matemáticos complejos del mundo real.

El tercer tipo de posicionamiento conceptual concibe las TIC como herramientas que deben ponerse al servicio de la consecución de las finalidades educativas. Por ello, resulta muy importante reflexionar alrededor de qué posibles usos educativos puede hacerse de cada tecnología para decidir cuál utilizar y cuándo, dónde y cómo usarla para enseñar y aprender de una forma más efectiva. Esta forma de concebir las TIC tiene su correlato conceptual con la noción de estrategia, caracterizada como un conjunto de procesos de toma de decisiones, intencionales, regulados y conscientes, que tienen como objetivo responder adecuadamente a la demanda de aprendizaje, usando el conocimiento disponible, y de forma ajustada a las características contextuales de la tarea propuesta (Monereo, Castelló, Clariana, Palma, y Pérez, 1994). Definidas de este modo, las estrategias de aprendizaje se sitúan en el nivel más alto de complejidad cognitiva, aparecen únicamente en procesos de aprendizaje vinculados a tareas abiertas y problemáticas, y son muy dependientes del contexto educativo.

Esta última característica, la contextualidad de toda estrategia de aprendizaje, supone que, concebida como un proceso de toma de decisiones que se transforman en acciones de los aprendices, se conforman en la práctica educativa en el aula como flujos complejos de

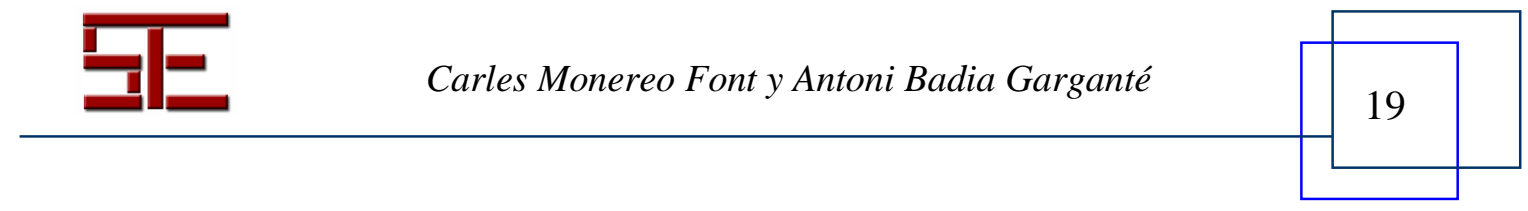




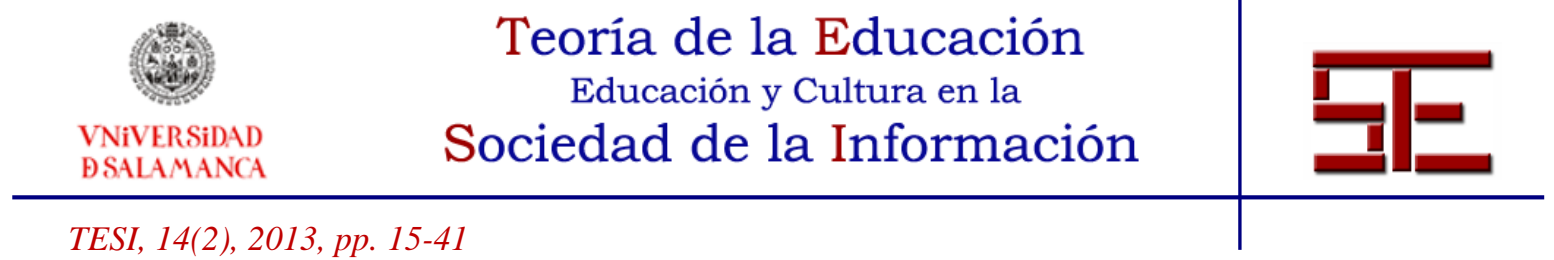

actos de aprendizaje, muy condicionados y dependientes del tipo de tarea de aprendizaje, del área y tipo de contenido curricular y, especialmente, del tipo de instrumentos mediadores usados para llevar a cabo dicha acción de aprendizaje. De este modo, las tecnologías de la información y la comunicación, consideradas como un tipo muy sofisticado de herramienta mediadora, poseen el poder de transformar los procesos de aprendizaje y la activación de las estrategias, relativos tanto a la toma de decisiones que se puede llevar a cabo, como al tipo y naturaleza de las acciones de aprendizaje de los alumnos.

\section{3.- CÓMO LAS TIC TRANSFORMAN LA FORMA DE COMPRENDER LAS ESTRATEGIAS DE APRENDIZAJE}

Seguidamente destacaremos siete aspectos característicos de las estrategias de aprendizaje que son transformados por el uso de las TIC en las aulas. En concreto, estos aspectos se refieren a la creación de nuevas situaciones y tareas educativas, a la asunción de nuevos retos en el aprendizaje, a una nueva caracterización del aprendizaje regulado, a la incorporación de procesos de regulación socialmente compartidos, a nuevas formas de acceder a los contenidos, a una explicación más compleja de los procesos cognitivos de aprendizaje, y a una ampliación de las formas de llevar a cabo los procesos de evaluación del aprendizaje del alumnado.

\subsection{Nuevas situaciones y tareas educativas}

Sería realmente costoso encontrar en la actualidad alguna actividad humana en la que no interviniesen, directa o indirectamente, las TIC. En el terreno educativo, aunque su potencial está infrautilizado, como han mostrado algunos estudios sobre su utilización en las aulas (ver la revisión de Coll, Mauri y Onrubia, 2008), su presencia e influencia aumentan de forma lenta, pero imparable, promoviendo y generando nuevas situaciones de enseñanza y aprendizaje que suponen nuevos desafíos para esos emigrantes digitales, en la afortunada metáfora de Prensky (2011), que somos los docentes atrapados entre la cultura impresa y la cultura digital. De forma esquemática, esos desafíos afectan a todo el proceso de transformación de los contenidos informativos en conocimiento utilizable, comunicable y evaluable. Cada una de las fases de este proceso se ha visto afectada por las TIC, desde la selección del contenido a enseñar, a sus modalidades de presentación, a las formas de negociación y apropiación de su sentido y significado, y a los sistemas de demostración y comunicación de lo aprendido. Por supuesto, esas nuevas situaciones no siempre han ido acompañadas de una nueva intencionalidad educativa; en la mayoría de los casos han obedecido a los supuestos epistemológicos y a las concepciones psicoeducativas

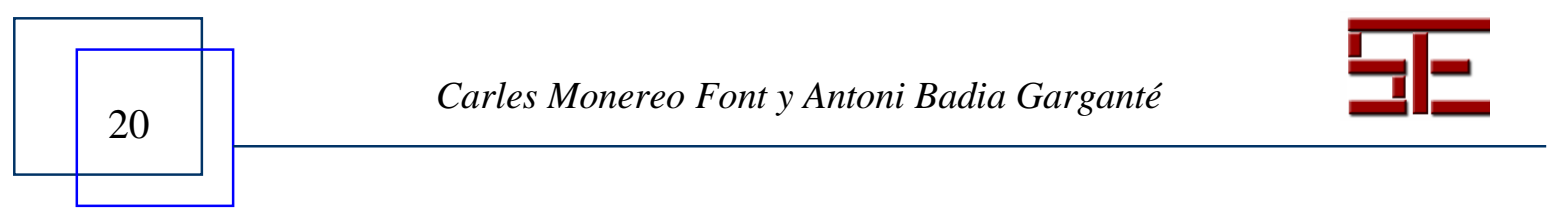




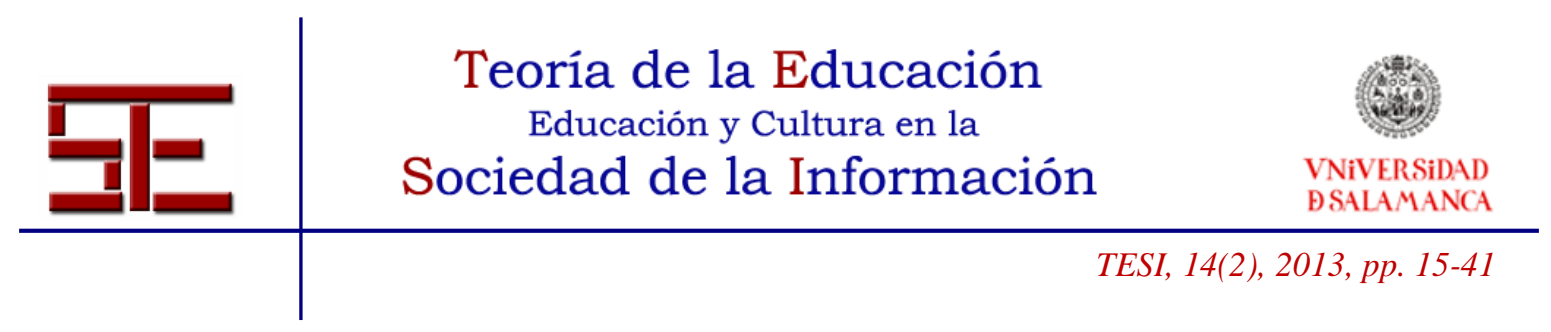

dominantes en cada colectivo e institución y, en consecuencia, han contribuido poco a una innovación real.

Sin embargo, y a pesar de las presiones centrípetas y conservadoras que caracterizan a las comunidades educativas, las TIC han actuado, en muchas ocasiones, como un caballo de Troya que ha abierto fisuras en los elementos más moleculares del sistema, las tareas y actividades educativas que usualmente se proponen a los alumnos. Las tradicionales tareas tipo ejercicio, poco contextualizadas, bien estructuradas, con instrucciones claras y precisas y de respuesta única, son fácilmente resolubles con la ayuda de las TIC. Claro que puede exigirse que se realicen sin el concurso de las TIC, por ejemplo, en situaciones de examen en el aula, sin embargo esta actitud resulta cada vez más anacrónica y menos argumentable: ¿se puede formar a ciudadanos, profesionales, investigadores para una sociedad de la información, evaluándoles sin tener en cuenta su dominio de las TIC?

La siguiente consecuencia es que, cuando se introducen tareas tipo problema, vinculadas a situaciones auténticas, y por lo tanto menos estructuradas, más ambiguas, abiertas y complejas, y que aceptan más de una respuesta correcta, el uso y adquisición de estrategias de aprendizaje se convierte en un requisito indispensable. Tal como ha señalado la investigación, este tipo de tareas en entornos virtuales (Inan, Lowther, Ross y Strahl, 2010; Scherer y Tiemann, 2012) necesitan y promueven la participación de estrategias de análisis de las condiciones de la demanda, de identificación, organización y regulación de las variables relevantes, de anticipación de los posibles resultados y de comunicación justificada de los mismos.

\subsection{Nuevos retos en el aprendizaje: currículo por competencias}

Aunque el concepto de competencia se sitúa en los años 70, es en la última década cuando la defensa de un currículo por competencias ha tenido un impacto a nivel planetario (Tiana, 2011). La práctica totalidad de los cinco continentes y todos los niveles educativos, desde pre-escolar hasta los estudios universitarios, se han visto impelidos a reestructurar sus planes de estudio y programaciones teniendo por objetivo la enseñanza de competencias. Aceptando el interés psicoeducativo del enfoque, no podemos obviar que detrás existen también intereses políticos y económicos derivados de la necesidad de homogeneizar los procesos formativos para facilitar la movilidad y la compatibilidad de los estudios, y vincular los aprendizajes a las necesidades laborales y sociales.

Desde nuestro punto de vista, una competencia sería un conjunto de conocimientos y estrategias aptas para resolver problemas relevantes dentro de un determinado sistema de actividad. La diferencia entre estrategia y competencia sería una cuestión de magnitud y relevancia del problema (Monereo y Pozo, 2001). La estrategia se orienta a la resolución de problemas más locales y acotados, mientras que la competencia requiere la coordinación de

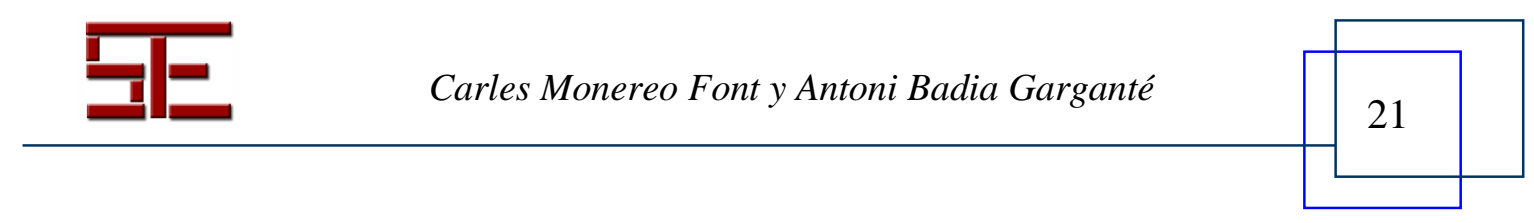




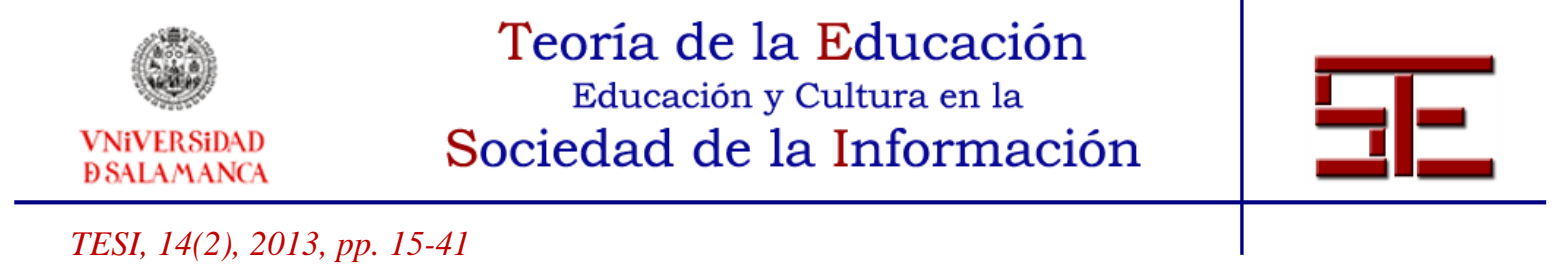

distintas estrategias y alude al afrontamiento de problemas complejos y significativos en un determinado ámbito de desarrollo. Estrategia en un psicólogo es realizar una entrevista, en un profesor preparar una clase sobre un tema concreto, en un investigador determinar el tipo de muestra más adecuada a su estudio; la competencia, respectivamente, es realizar un diagnóstico completo de un caso, programar una asignatura, desarrollar de forma coherente una línea de investigación. Desarrollando esta lógica argumental, el aprendizaje de una competencia requeriría la adquisición de algunas estrategias, que a su vez demandaría la presencia y activación de un conjunto de conocimientos apropiados al problema que pretenda resolverse.

Para que una enseñanza y evaluación centrada en competencias resulte genuina debe abordarse en base a problemas auténticos. Analicemos brevemente esos términos. Cuando hablamos de problemas nos referimos a situaciones para cuya resolución se precisa un comportamiento autorregulado (en oposición a "ejercicio", distinción ya abordada en un apartado anterior). Nos parece interesante distinguir problemas prototípicos o frecuentes de aquellos que aún no lo son pero que presumiblemente lo serán a medio plazo: problemas emergentes (Monereo y Badia, 2012). En cuanto a la autenticidad, en la literatura se han señalado cuatro componentes básicos que determinan el grado de autenticidad de un problema (Gulikers, Bastiaens y Kirschner, 2004): su nivel de realismo (fidelidad con el problema en condiciones reales), de relevancia (representatividad respecto a los problemas cruciales en un ámbito de desarrollo), de autorregulación (exigencia en la planificación, supervisión y evaluación de la resolución) y de socialización (incorporación de expresiones, herramientas, formatos de interacción, etc., propios de la comunidad de prácticas en la que suele inserirse el problema).

Como distintos trabajos han mostrado (Badia y Monereo, 2008; Monereo, 2005; Stein, Isaacs y Andrews, 2004), las TIC son un medio idóneo para trabajar con problemas auténticos y, por lo tanto, para promover el aprendizaje y la evaluación de estrategias y competencias. Sin que pretendamos agotar todas las posibilidades, la red de redes puede promover la autenticidad al menos a partir de tres vías:

1. Mediante el acceso directo a fuentes primarias de información, a veces incluso en el mismo momento en que se está produciendo un fenómeno (por ejemplo, mediante el visionado en directo de una cámara que graba un determinado acontecimiento), sin que existan filtros para su análisis e interpretación. Ello permite a los estudiantes actuar como una agencia de noticias y procesar la información "en crudo" en todas sus fases.

2. Mediante sistemas de emulación basados en inteligencia artificial (Monereo y Romero, 2008).

3. A través de la resolución de casos, problemas y/o proyectos que respondan a situaciones sociales reales, de conflicto o apuro, que necesiten de una respuesta solidaria o cívica por parte de la comunidad. Investigadores como Herrington y Kervin (2007), y más recientemente Neo, Neo y Tan (2012), nos presentan excelentes ejemplos de integración curricular de las TIC para afrontar problemas auténticos (por ejemplo, cómo evitar la molesta presencia de

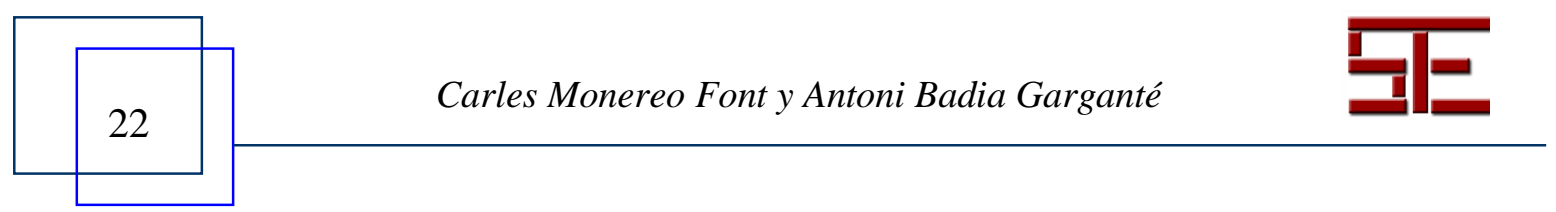




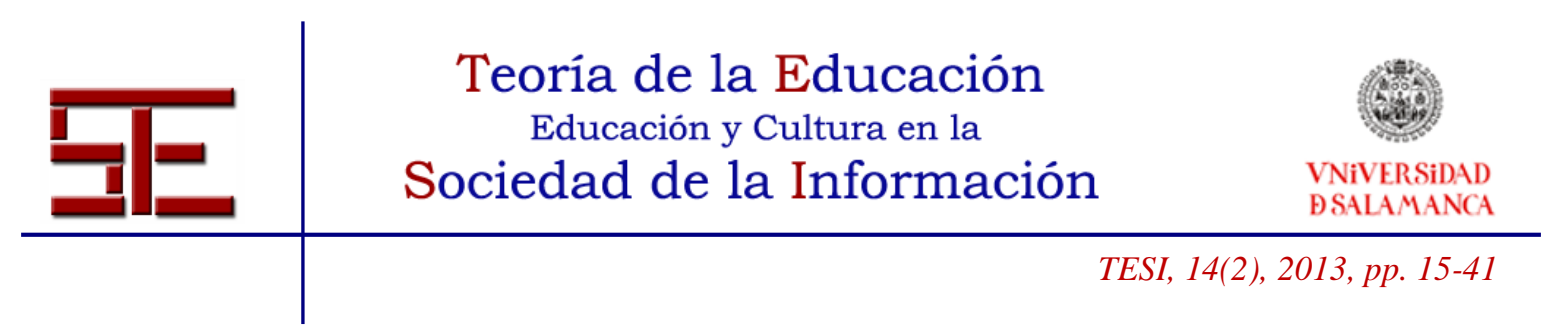

mosquitos y ratas en un parque muy popular en el que los turistas suelen dejar restos de comida), en el que los estudiantes deben utilizar múltiples estrategias de aprendizaje (toma de notas, recolección y categorización de evidencias, elaboración de informes, preparación de exposiciones orales, etc.) para gestionar contenidos correspondientes a áreas curriculares diversas (ciencias sociales, ciencias naturales, lenguaje y matemáticas).

\subsection{El aprendizaje autorregulado y corregulado mediante las TIC}

La autorregulación del aprendizaje hace referencia a los procesos individuales mediante los cuales los alumnos activan, orientan y mantienen sus pensamientos, afectos y acciones hacia la consecución de objetivos de aprendizaje. Estos procesos consisten básicamente en que el alumno utiliza "bucles autogenerados de información" que le permiten ir ajustando progresivamente sus pensamientos, afectos y acciones, para que así estén dirigidos siempre de forma adecuada a la consecución de los objetivos de la tarea de aprendizaje. Los estudiantes que autorregulan su aprendizaje habitualmente también saben establecer metas razonables de aprendizaje, aplicar los conocimientos disponibles, desplegar estrategias de aprendizaje efectivas, monitorizar y evaluar el progreso hacia el objetivo de aprendizaje, y modificar las condiciones del contexto educativo a las metas de aprendizaje (Zimmerman y Schunk, 2011). La autorregulación del aprendizaje debe entenderse como un conjunto de procesos específicos de planificación, monitorización y evaluación de la propia actuación propios de tareas de aprendizaje específicas y prototípicas de distintas áreas de contenido curricular específico, tales como las matemáticas, la lectura, la escritura, las ciencias o la historia, entre otras. Según Hadwin, Järvelä y Miller (2011), la corregulación del aprendizaje se produce cuando un alumno necesita coordinarse temporalmente con otros para conseguir los objetivos formulados, y se desarrolla mediante interacciones que influyen en el proceso de autorregulación.

Las tecnologías transforman los procesos de autorregulación y corregulación del aprendizaje al menos en tres niveles diferentes: a) Conformando la autorregulación mediante indicaciones reflexivas (reflection prompts); b) Aportando feedback en relación al proceso de aprendizaje; y c) Mediante la interacción con un "metatutor".

Para que el alumno pueda resolver una tarea de aprendizaje con altos niveles de autorregulación del aprendizaje las TIC pueden contribuir aportando indicaciones reflexivas insertadas de forma contextualmente significativa en "entornos de aprendizaje basados en web". Dichas indicaciones, que han sido elaboradas previamente a la realización de la tarea y también se presentan en formatos diseñados tecnológicamente, funcionan como señales que provocan la reflexión del alumno facilitando de este modo

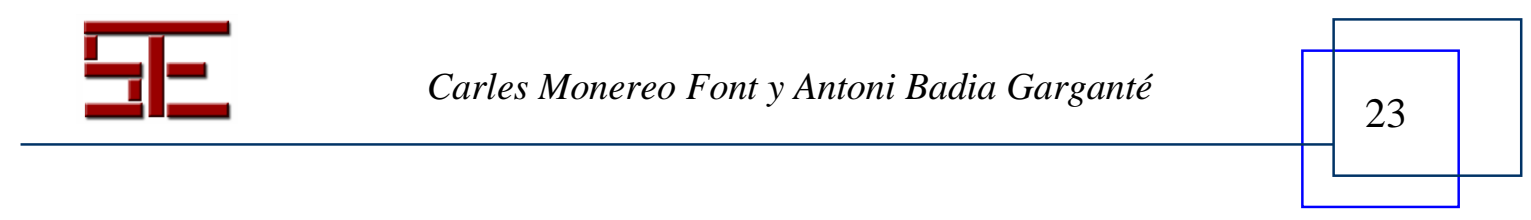




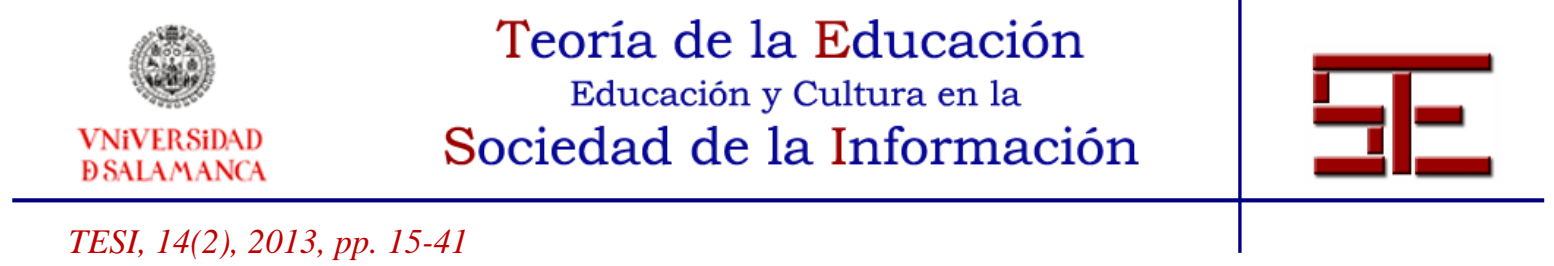

los procesos de planificación, supervisión y evaluación del propio proceso de aprendizaje (Azevedo y Hadwin, 2005; Van den Boom, Paas, Van Merrienboer y Van Gog, 2004).

Otra forma de intervención de las TIC en la autorregulación del aprendizaje se puede producir facilitando el acceso del estudiante al feedback sobre su trabajo por parte del profesor o de sus compañeros (Van der Boom, Paas y Van Merriënboer, 2007). El estudiante puede tener acceso a ambos tipos de feedback, entre otras vías, mediante mensajes insertados en el propio entorno electrónico (referidos a aspectos del contexto de aprendizaje), en los documentos de trabajo (referidos a los procesos de planificación, supervisión o evaluación del trabajo realizado) o también mensajes de correo electrónico con un carácter más general.

El uso de un metatutor puede considerarse la forma más avanzada, desde el punto de vista tecno-pedagógico, de ayuda a los alumnos en los procesos de regulación (Azevedo, Johnson, Chauncey y Graesser, 2011). En el ejemplo presentado por estos autores, un metatutor es un entorno hipermedia de aprendizaje que detecta, rastrea, modeliza y fomenta la autorregulación del aprendizaje de los contenidos curriculares de los sistemas de funcionamiento del cuerpo humano. Este metatutor incluye 13 diferentes procesos, tres relacionados con la planificación del aprendizaje (crear planes, proponer sub-objetivos y activar los conocimientos previos), cuatro relativos a la supervisión del proceso de aprendizaje (juzgar el aprendizaje, sentir que se va adquiriendo conocimiento, evaluar el contenido y supervisar el proceso hacia la consecución de los objetivos) y seis que hacen referencia a los procesos cognitivos implicados en la resolución de la tarea de aprendizaje (coordinar las fuentes de información, resumir, releer, dibujar, tomar notas y realizar inferencias).

\subsection{Los procesos de regulación socialmente compartida a través de la tecnología}

Para Hadwin, Järvelä y Miller (2011), la regulación socialmente compartida del aprendizaje se refiere a los procesos regulativos colectivos, organizados y dirigidos a la consecución de un objetivo grupal de aprendizaje. Considerados únicamente en tareas de aprendizaje colaborativo, dan cuenta del modo en que un grupo de aprendizaje, definido como un sistema unitario, se autorregula grupalmente para desarrollar procesos compartidos de planificación, supervisión y evaluación de la actividad de aprendizaje.

Las tecnologías que, a día de hoy, mejor pueden apoyar los procesos grupales de regulación socialmente compartida son las denominadas herramientas de consciencia de

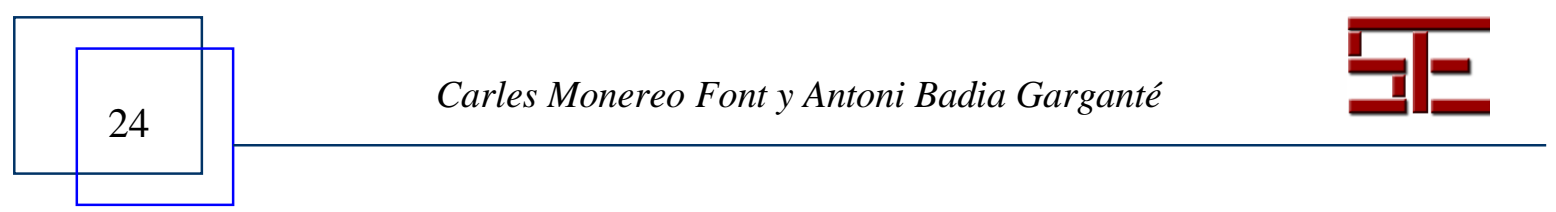




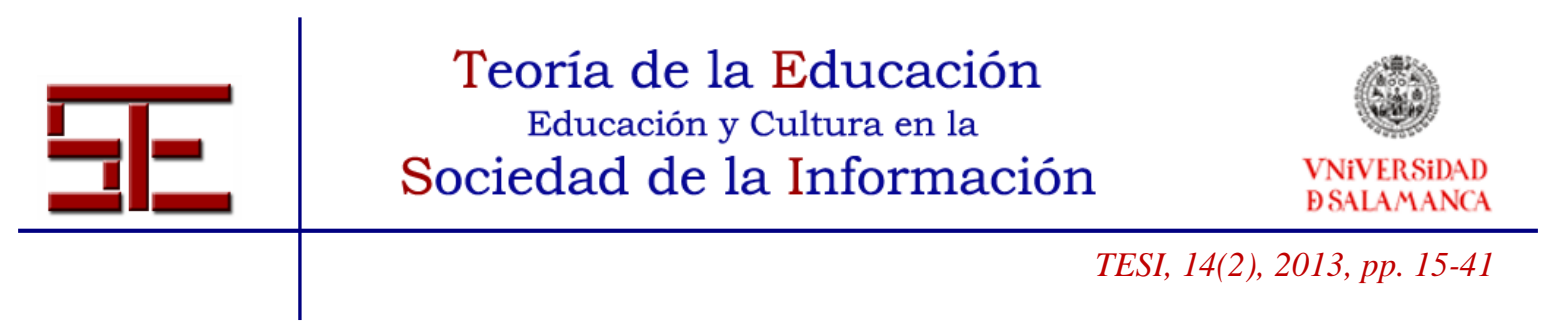

grupo (Janssen, Erkens, y Kirschner, 2011). Estas herramientas tienen como objetivo incrementar el conocimiento grupal sobre la dinámica colaborativa del propio grupo, para de este modo favorecer la coordinación de las acciones de sus miembros.

Según Janssen y Bodemer (2013), en el aprendizaje colaborativo basado en el uso de ordenadores pueden identificarse dos tipos de conciencia de grupo, que denominan conciencia cognitiva grupal y conciencia social grupal. La conciencia cognitiva grupal se promueve proporcionando y compartiendo información sobre el conocimiento y la experticia de los miembros del grupo. La conciencia social del grupo se fomenta proveyendo información sobre las contribuciones de los miembros al proceso de aprendizaje del grupo. Ello es posible porque una de las características de las tecnologías, especialmente de los entornos tecnológicos de aprendizaje grupal basados en la comunicación asíncrona y escrita, consiste en la posibilidad de acceder a toda la información, registrada en la plataforma, relativa al proceso de interacción educativa entre los miembros. Esta información puede referirse tanto a los mensajes que se han intercambiado los miembros como al proceso de elaboración conjunta de documentos y al propio proceso de escritura.

El hecho de compartir ambos tipos de información por medio de herramientas tecnológicas específicamente creadas para ello afecta el modo en que los miembros del grupo abordan el aprendizaje de los contenidos y el mantenimiento de un clima grupal positivo.

\subsection{El acceso a los contenidos digitales}

A pesar de la importancia capital de ser un buscador y "encontrador" competente (un gatekeeper en su acepción inglesa), sufrimos un proceso de "googlelización" creciente según el cual el $60 \%$ de las búsquedas que se efectúan en Internet emplean ese motor como único sistema de búsqueda (Monereo, 2009). Las investigaciones que se han realizado sobre los procedimientos de búsqueda que utilizan los estudiantes (básicamente de secundaria y universitarios) coinciden en señalar que su competencia presenta múltiples carencias. Por resaltar las más destacadas (Hirata y Hirata, 2010; Monereo y Fuentes, 2008):

- Utilizan palabras clave extraídas directamente de la demanda o enunciado de la búsqueda dada por el profesor.

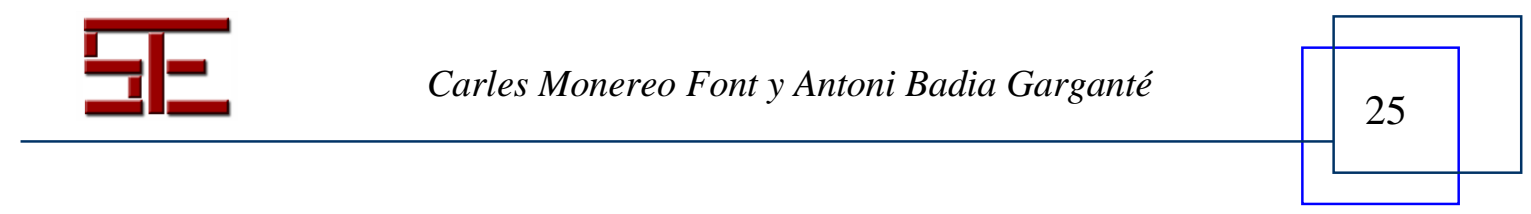




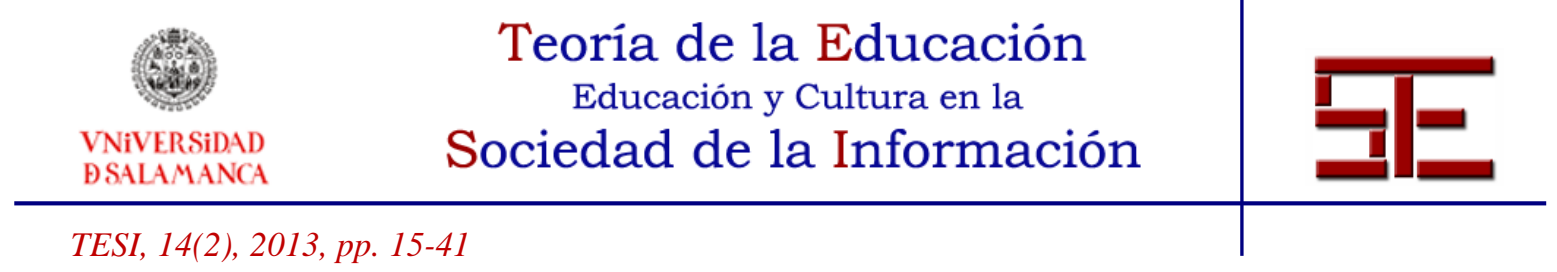

- Evalúan la fiabilidad de una página web atendiendo más al diseño visual o a información curiosa que a indicadores que aporten credibilidad sobre los contenidos incluidos.

- En relación al procesamiento de lo encontrado, muestran dificultades en la lectura de páginas web para determinar su pertinencia. Muchos leen palabra por palabra y les resulta difícil obtener una comprensión global del sitio.

- En realidad no suelen juzgar la validez de la información y son poco selectivos en la elección de fuentes y en la información que finalmente adoptan. Suelen conformarse con una comprensión superficial de lo que leen.

- Difícilmente realizan una nueva búsqueda, modificando los parámetros y aprendiendo de los errores cometidos.

Parece evidente que, a pesar de formar parte de la generación de nativos digitales, necesitan una formación específica que les enseñe un modelo de búsqueda eficaz y eficiente y les proporcione herramientas para aprender a buscar y seleccionar contenidos relevantes. A partir de distintos modelos de búsqueda, originados tanto desde los estudios documentales y biblioeconómicos, como desde la psicología cognitiva, se han realizado algunas propuestas integradoras del conjunto de toma de decisiones que requeriría una búsqueda estratégica (Monereo, 2009; Monereo y Fuentes, 2008), a saber: a) examen de la consulta o demanda de búsqueda a realizar; b) planificación de la búsqueda, con especial énfasis en los distintos movimientos, tácticas y estrategias de búsqueda a implementar; c) monitorización y supervisión de la búsqueda, regulando las siguientes decisiones en función del ajuste de lo encontrado a la demanda, respecto a la calidad de lo hallado y en cuanto a su fiabilidad; d) evaluación del resultado de la búsqueda a través de la integración y elaboración de lo encontrado en un único documento; e) evaluación del propio proceso de búsqueda con el fin de aprender de los posibles errores cometidos y f) cumplimentación final de la tarea o consulta que ha originado la búsqueda.

Con respecto a este modelo estándar, es preciso realizar dos consideraciones. En primer lugar su aprendizaje debe realizarse en base a situaciones de formación adaptadas a las características de los aprendices. Actualmente existen múltiples alternativas para formar a los estudiantes, de cualquier nivel educativo, en estrategias de búsqueda. Dichas propuestas podrían agruparse atendiendo al nivel de protagonismo y complejidad en la toma de decisiones que otorgan al alumno. En este sentido existirían propuestas de búsqueda cerradas, en las que existe una pre-selección de qué buscar y dónde hacerlo,

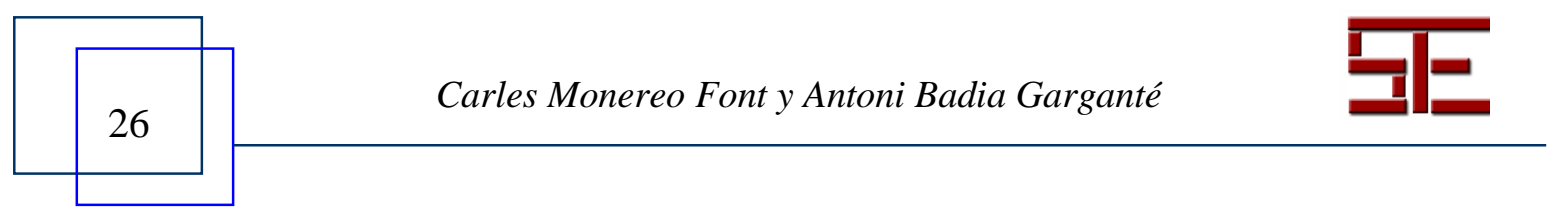




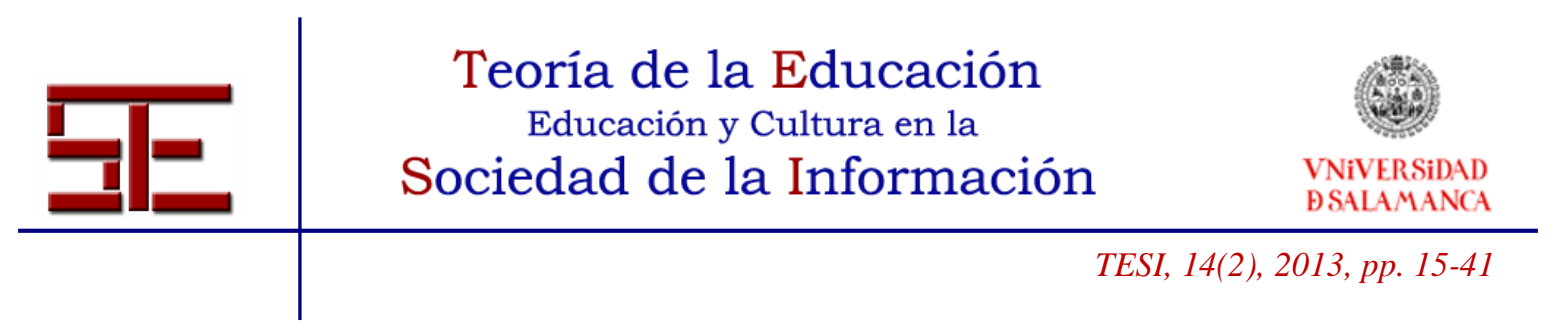

como ocurre con los collage multimedia (Scarpbook), la caza del tesoro (Treasure Hunter) o las guías didácticas de navegación (Webquest); otras que permiten una exploración más libre de los estudiantes dentro de un espacio virtual amplio (simulación de un museo, empresa, laboratorio, etc.), como los denominados viajes virtuales (Virtual Field Trips), y, finalmente, propuestas en que son los propios alumnos quienes diseñen la búsqueda, formulan preguntas que implican diferentes niveles cognitivos de exigencia y recomiendan distintos itinerarios de búsqueda (Du Plessis y Webb, 2011).

La segunda consideración que señalábamos consiste en recordar que ese modelo de búsqueda (como cualquier otro que se adopte) debe emplearse de forma también estratégica, es decir, flexible, en función siempre de las condiciones y objetivos de búsqueda (Monereo y Badia, 2012). Por ello, en ocasiones, será adecuado:

1) Utilizar el denominado "patchworking (realizar una mezcla o un pastiche), que incluye desde la copia e inserción literal de un texto en otro, hasta una verdadera intertextualización significativa del nuevo texto, de forma que pueda llegar a producir una nueva idea.

2) Saltarse algunos pasos innecesarios, o re-iniciar un nuevo ciclo de búsqueda o demorar el procesamiento de una información para más adelante.

3) Dedicar una mayor atención y esfuerzo de regulación en aquellas fases en las que, como hemos visto, algunos estudiantes encuentran mayores dificultades, o que requieran, por las condiciones de búsqueda (por ejemplo preparación de un examen estresante), un especial estado de concentración y control emocional.

\subsection{Los procesos cognitivos de aprendizaje}

El hecho de que los procesos mentales de un individuo no puedan ser comprendidos como un fenómeno cognitivo que sucede únicamente en su cabeza ocasiona un replanteamiento importante sobre la influencia que pueden tener las TIC sobre el funcionamiento mental humano y, en particular, sobre los procesos cognitivos individuales de aprendizaje. Sin ánimo de ser exhaustivos, seguidamente indicamos dos tipos de influencia, a modo de ejemplos relevantes.

La primera influencia destacable tiene que ver con la necesidad del sistema cognitivo humano de adaptarse al elevado nivel de formalismo que poseen las funciones y operaciones que pueden ejercerse mediante los programas informáticos. A estas alturas

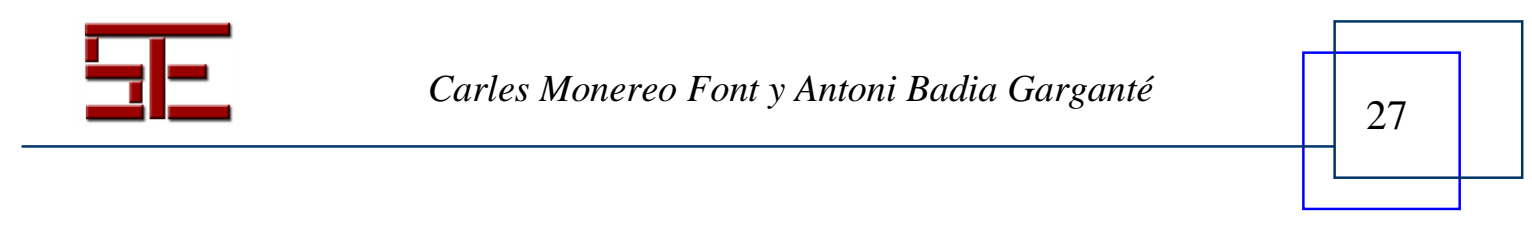




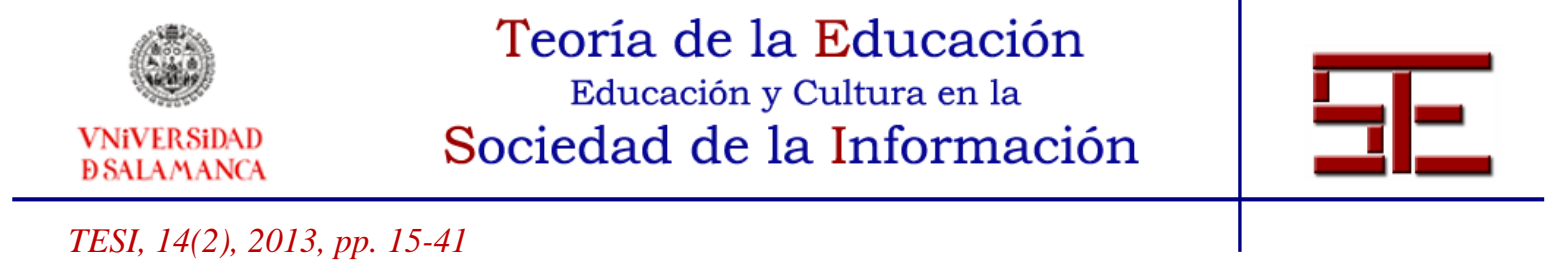

resulta innecesario constatar las diferencias en los procesos cognitivos desplegados en la escritura mediante papel y lápiz, o los procesos cognitivos que deben activarse al escribir, utilizando las posibilidades que brindan actualmente los procesadores de textos.

La segunda influencia se refiere a las aportaciones que realizan las tecnologías al funcionamiento cognitivo humano. Varios autores clásicos de referencia (Pea, 1985; Salomon, 1985; Salomon, 1992; Salomon, Perkins y Globerson, 1992) señalan dos tipos de impacto.

En primer lugar, las tecnologías pueden ser referentes en el modo de realizar operaciones $\mathrm{y}$ funciones en determinadas tareas, $\mathrm{y}$ el aprendiz puede internalizar estas herramientas tecnológicas simbólicas y, de este modo, apropiárselas y convertirlas en herramientas cognitivas. Ello será posible siempre y cuando el aprendizaje se mueva dentro de la zona de desarrollo próximo del aprendiz.

En segundo lugar, las tecnologías pueden asociarse con el aprendiz, de modo que le permitan amplificar o reorganizar los procesos de pensamiento cuando está resolviendo una tarea de aprendizaje. En el primer caso, las tecnologías posibilitan realizar las tareas habituales de aprendizaje de manera más eficiente y en menos tiempo. Comúnmente, ello se consigue porque el ordenador realiza aquellas partes de las tareas de bajo nivel, operativas y automatizables, y por ello el aprendiz puede dedicarse a las partes de las tareas que demandan el despliegue de habilidades de orden superior. En el segundo caso, el ordenador posibilita la realización de nuevas tareas de aprendizaje, que no sería posible realizar sin la contribución de la tecnología, de modo que ésta tiene la capacidad de redefinir y reestructurar el funcionamiento humano en el momento de aprender.

\subsection{Los procesos de evaluación del aprendizaje del alumno}

La evaluación del aprendizaje con intervención de las tecnologías ha sido en los últimos años uno de los campos de conocimiento que más ha avanzado. Según Redecker y Johannessen (2013), nos encontramos en la antesala de lo que los autores consideran las formas futuras de diseñar las estrategias de evaluación, y que denominan el enfoque de la evaluación integrada. Todas las investigaciones realizadas en los últimos años indican que cuanto más y mejor feedback reciba el estudiante en relación con su actividad de aprendizaje, más aumentará la motivación, la calidad de los procesos de autorregulación, las decisiones que el alumno tome en su aprendizaje y su rendimiento académico.

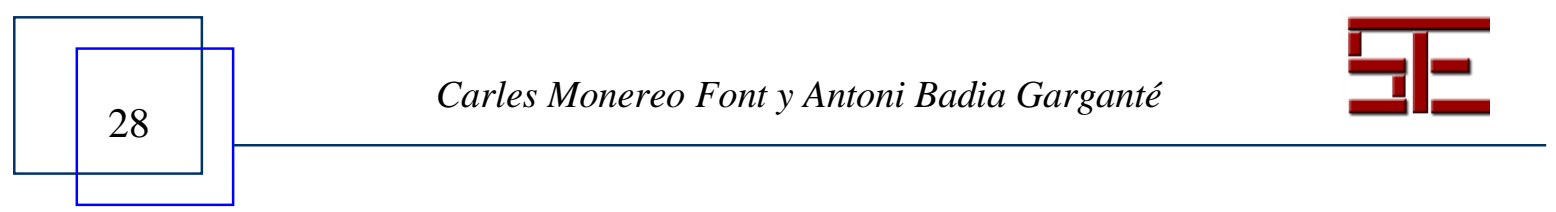




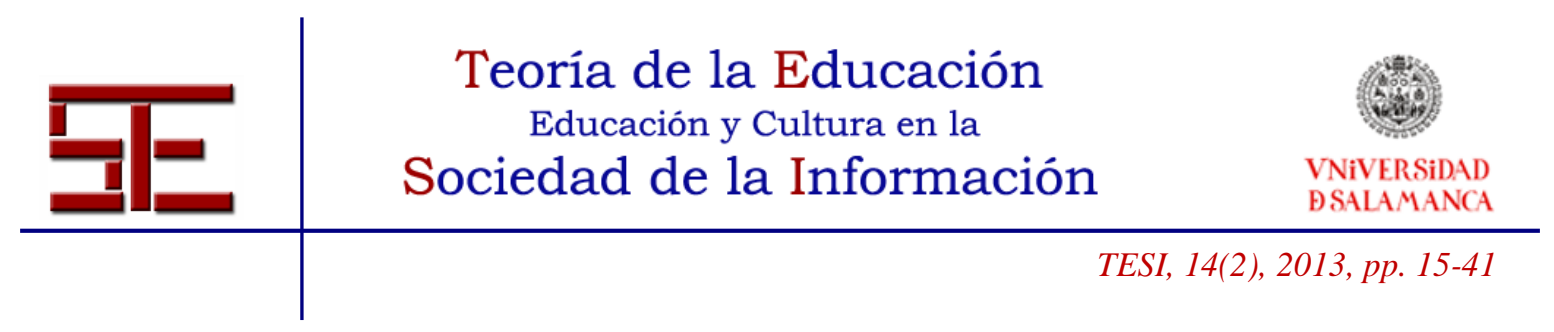

En síntesis, este enfoque se basa en la posibilidad de que las tecnologías puedan aportar al profesor un flujo permanente de información real sobre las necesidades y el progreso individual de cada aprendiz. Y, con esta información, y en base a los principios educativos derivados de la evaluación formativa, el profesor (o una aplicación tecnológica que funciona como un profesor) puede intervenir para personalizar las ayudas educativas que necesita el aprendiz mediante una tutoría individual.

Este bucle formado por tres procesos encadenados relacionados con el aprendizaje de los alumnos y la evaluación formativa (obtención de información, valoración de la información, retorno de la información) puede hacerse por medio de diversos niveles de automatización. La obtención de la información sobre el progreso en el aprendizaje es quizá el proceso que puede automatizarse mejor y, por consiguiente, puede llegar a ser llevado a cabo por la tecnología. Por ejemplo, ya existen programas denominados "sistemas tutoriales inteligentes" que pueden proporcionar información cualitativa sobre por qué determinadas respuestas de los alumnos son incorrectas (Nunan, 2010), sobre los patrones de aprendizaje desplegados (Looney, 2010) o sobre la calidad de la estructura de los argumentos científicos que utilizan los alumnos (Huang et. al, 2011).

Los programas informáticos son usados también en numerosas ocasiones para valorar la información recogida. Habitualmente este proceso se realiza por comparación, entre la ejecución del alumno y un conjunto de modelos de ejecución o de respuesta esperados. Este es el proceso de mayor dificultad de automatización, puesto que el programa únicamente podrá comparar actuaciones de los alumnos que tenga almacenadas previamente en forma de modelos de ejecución o de respuestas correctas. Los programas más comunes suelen valorar grados de respuesta o ejecución de los alumnos.

Esta valoración llevada a cabo por el programa suele traducirse en diversas modalidades de feedback automático, que se concreta por ejemplo mediante la adaptación del nivel de la dificultad de las tareas de aprendizaje al progreso y las necesidades de aprendizaje de cada alumno (Ljungdahl y Prescott, 2009).

Acabamos de ver el modo en que estas siete características básicas de la definición de estrategia de aprendizaje han variado su significado cuando se ha tenido en cuenta la contribución de las TIC. Seguidamente ejemplificaremos algunos de estos cambios aplicados a las tareas de resolución de problemas informacionales.

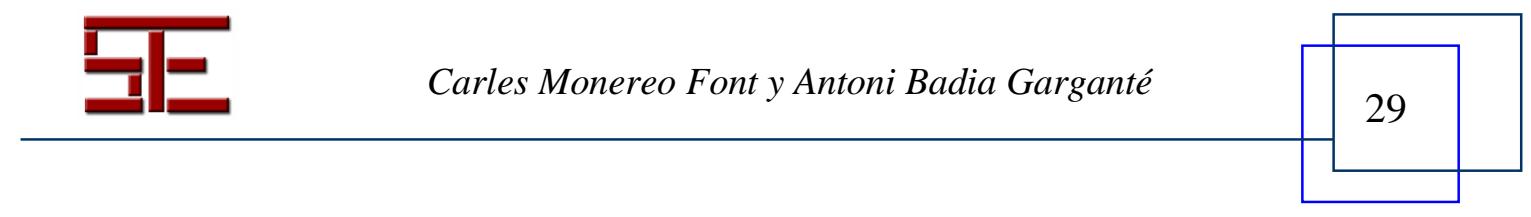




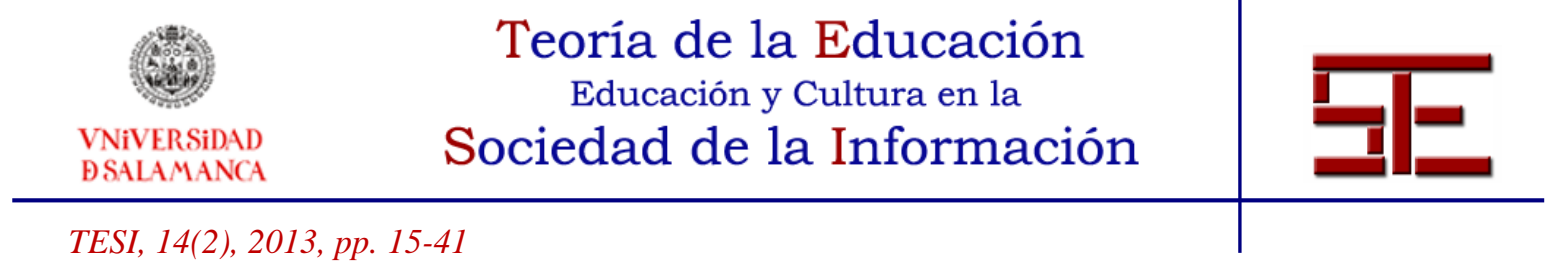

\section{4.- APRENDIZAJE ESTRATÉGICO Y USOS EDUCATIVOS DE LAS TIC EN TAREAS DE RESOLUCIÓN DE PROBLEMAS INFORMACIONALES EN HISTORIA}

Tal como acabamos de indicar, las tecnologías de la información y la comunicación, y los usos que de ellas se hacen en contextos educativos, han transformado de manera profunda la forma de concebir las estrategias de aprendizaje. También hemos explicado que esta transformación se produce en el marco de tareas de aprendizaje situadas contextualmente y con contenidos curriculares específicos. A modo de ejemplificación, seguidamente expondremos cómo concebiríamos el aprendizaje estratégico en una tarea de resolución colaborativa de problemas informacionales en la disciplina de la Historia, usando plataformas educativas de comunicación asincrónica y escrita (Badia, Becerril y Romero, 2010). En concreto, la tarea que nos proponemos ejemplificar consiste en que varios grupos de alumnos de cuarto curso de Educación Secundaria Obligatoria traten de responder a la siguiente pregunta: ¿hasta qué punto fue beneficiosa o perjudicial, para los primeros habitantes del territorio conocido actualmente como México, la conquista y posterior colonización por parte de los españoles? Los estudiantes deben responder a la pregunta escribiendo un informe de forma colaborativa. De hecho, consiste en un proyecto de aprendizaje en colaboración entre un aula de un centro educativo español y una de un centro educativo mexicano y, por lo tanto, cada grupo estará conformado por 2-3 alumnos mexicanos y 2-3 alumnos españoles. Pueden desarrollar dicho proyecto colaborativo durante 8 semanas.

Se trata de resolver un problema informacional en el campo de la enseñanza de la Historia, puesto que se caracteriza por ser una tarea compleja de aprendizaje que debe ser resuelta por unos alumnos que no poseen, de entrada, toda la información histórica necesaria para su resolución (Becerril y Badia, en prensa; Monereo y Badia, 2012), y además no existe ni un único proceso para su resolución, ni una solución única. Por el contrario, resulta más apropiado hablar de mejores o peores procesos de resolución del problema informacional, así como de diversos grados de calidad de la respuesta de los alumnos. Ante una tarea de esta naturaleza, ¿de qué modo se interrelacionan el aprendizaje estratégico y los usos educativos de las TIC? ¿Cómo se produce el proceso de toma de decisiones cuando se trata de un aprendizaje online?

En primer lugar, debe tenerse en cuenta que las TIC posibilitan que se pueda proponer una demanda de alta complejidad en cuatro aspectos que tienen implicaciones directas con las decisiones que debe tomar un aprendiz. El primer aspecto que añade complejidad

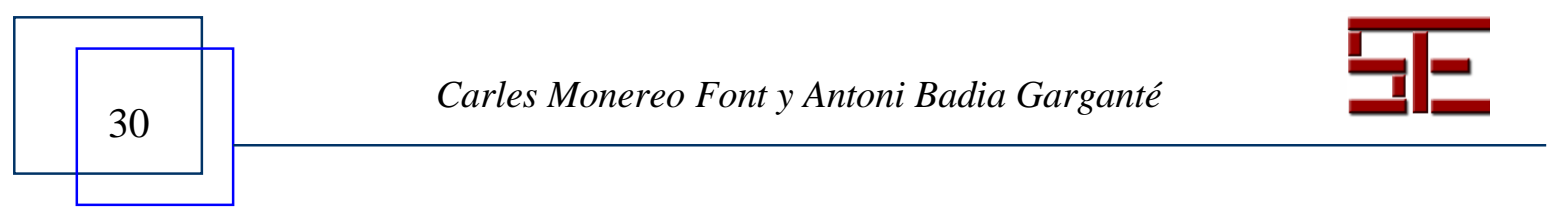




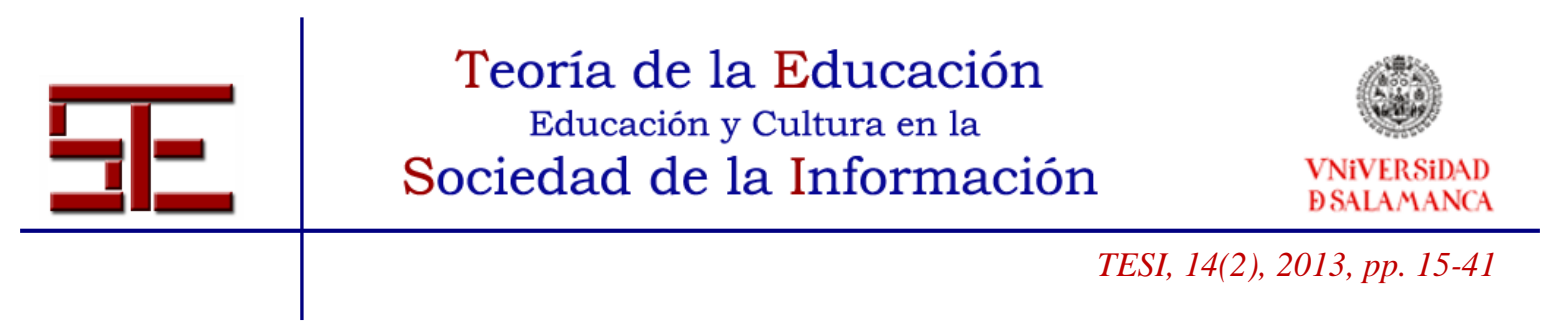

es que se trata de una actividad de aprendizaje poco estructurada, en donde puede haber múltiples vías de resolución y varias respuestas que podrían considerarse adecuadas. El segundo aspecto tiene que ver con que los alumnos son requeridos a poner en juego, de manera apropiada, al menos dos competencias básicas, como son la competencia informacional (en relación con la información) y la competencia colaborativa (en relación con la interacción educativa con los compañeros). Además, se trata de una tarea que requiere, para su correcta resolución, del manejo de una cantidad muy amplia de información y de un alto grado de coordinación entre las contribuciones de los miembros de un grupo. Otro aspecto que añade mayor complejidad se refiere al alto grado de exigencia en cuanto a las habilidades de aprendizaje que deben desplegarse (puesto que requerirá niveles altos de elaboración y comunicación de la información entre los aprendices) y a la necesidad de activar adecuadamente los procesos de autorregulación y, sobre todo, de corregulación del aprendizaje dentro de cada grupo.

Tal como la tarea está planteada, las TIC cambian cuatro condiciones que caracterizan una tarea similar de aprendizaje desarrollada sin la participación de las TIC. En primer lugar, las aulas virtuales (Barberà y Badia, 2005) hacen posible que la tarea de aprendizaje pueda llevarse a cabo sin necesidad que los participantes compartan un mismo espacio geográfico. Este aspecto es muy relevante puesto que, como sucede en la tarea planteada, varios estudiantes, distantes geográfica y culturalmente, pueden ser miembros de un mismo grupo virtual de aprendizaje. En segundo lugar, las aulas virtuales también posibilitan distintas modalidades de comunicación entre los miembros de un grupo, destacando en especial la comunicación escrita, que posee algunas características muy relevantes para los procesos educativos. En tercer lugar, la tarea también requerirá un manejo diferente del tiempo educativo, tanto en lo concerniente a la asincronía en la comunicación entre los miembros como en los tiempos de respuesta de los mensajes. Y, por último, también deberá tenerse en cuenta que los miembros del grupo podrán hacer uso de multitud de herramientas colaborativas referidas al acceso a la información, y a la construcción conjunta de conocimiento por medio, por ejemplo, de herramientas de escritura colaborativa.

Resulta frecuente que los alumnos sin experiencia previa en este tipo de tareas de aprendizaje virtual tengan dificultades tanto en la comprensión adecuada de la demanda como en la interpretación apropiada de las condiciones del contexto de resolución de la demanda informacional (Monereo y Badia, 2012).

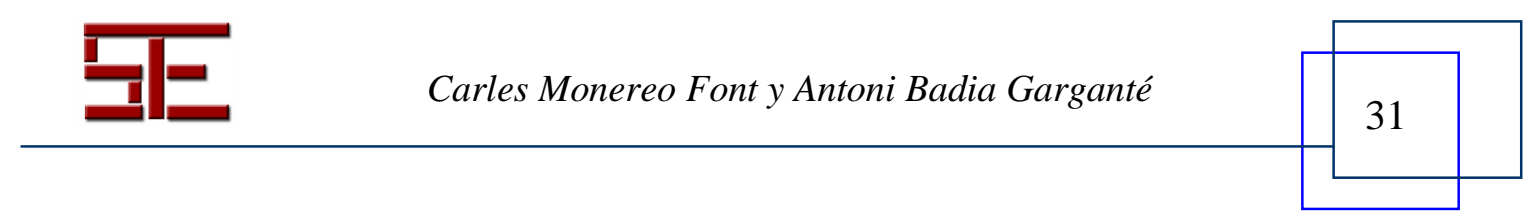




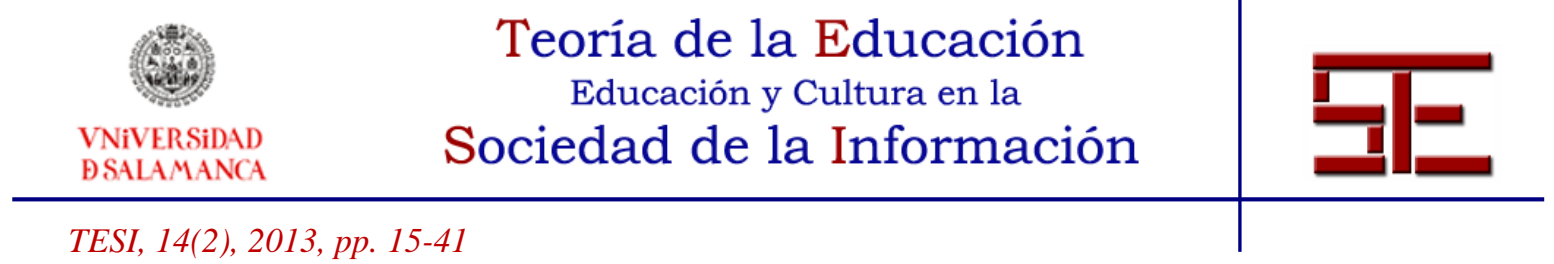

La tecnología también puede contribuir al despliegue del aprendizaje estratégico de los alumnos guiando las decisiones que toman respecto a tres aspectos: la gestión de la información de contenido, la interacción educativa entre iguales y los procesos de resolución de la tarea informacional, mediante los denominados guiones de aprendizaje (en inglés, learning scripts). Un guion de aprendizaje está constituido por un conjunto de orientaciones y recomendaciones que facilitan el desarrollo de un determinado proceso de aprendizaje. Pueden ser facilitados por el profesor o por la propia tecnología. Existen al menos tres tipos de información que puede ser proporcionada por medio de guiones de aprendizaje, relacionados con los tres aspectos anteriores, que algunos autores (Weinberger, Ertl, Fischer y Mandl, 2005) denominan: guiones epistémicos, guiones colaborativos y guiones de la resolución de la tarea informacional.

Los guiones epistémicos orientan las actividades de construcción de conocimiento, guiando a los aprendices hacia aspectos específicos de la tarea y de los contenidos para favorecer de este modo la asimilación de nuevo conocimiento, la integración de nuevo conocimiento a los conocimientos previos y la construcción de conocimiento compartido. Los guiones colaborativos (Fischer, Kollar, Stegmann y Wecker, 2013) facilitan el aprendizaje entre iguales guiando y coordinando la realización de las actividades colaborativas más apropiadas para la consecución de los objetivos perseguidos. Ello se consigue especificando qué actividades colaborativas son, en qué secuencia deben realizarse y asignando qué tareas deben realizarse grupalmente y cuáles deben desarrollarse individualmente. Por último, los guiones relacionados con la resolución de la tarea informacional (Raes, Schellens, De Wever y Vanderhoven, 2012) guían a los estudiantes en el proceso de resolución del problema informacional, focalizando en cuatro aspectos regulativos del aprendizaje (orientación, monitorización, manejo y evaluación de la actividad de aprendizaje) relacionados con cinco procesos relativos a los contenidos (definir el problema informativo, buscar información, analizar la información, procesar la información, y organizar y presentar la información).

\section{5.- RETOS FUTUROS EN LA INVESTIGACIÓN SOBRE EL APRENDIZAJE ESTRATÉGICO}

En un terreno tan sujeto a cambios como el de las TIC, resulta cuando menos arriesgado realizar previsiones sobre cuáles serán los futuros puntos de mira en los que se concentrará la investigación. Dicho esto, si revisamos las publicaciones del Social Sciences Citation Index del último lustro, en las que se cruzan los tópicos de estrategias

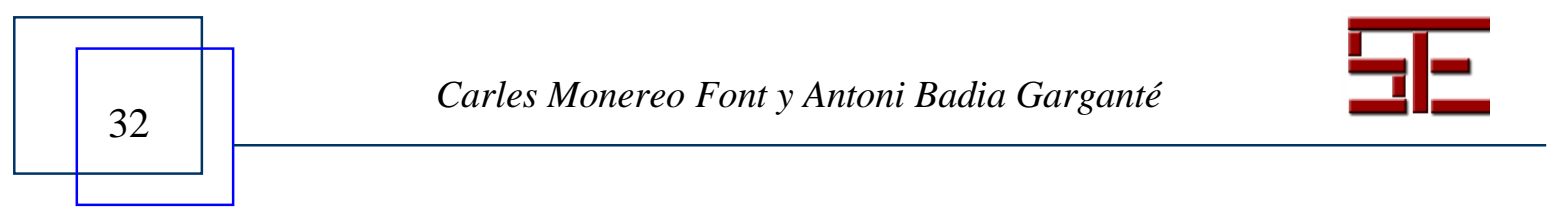




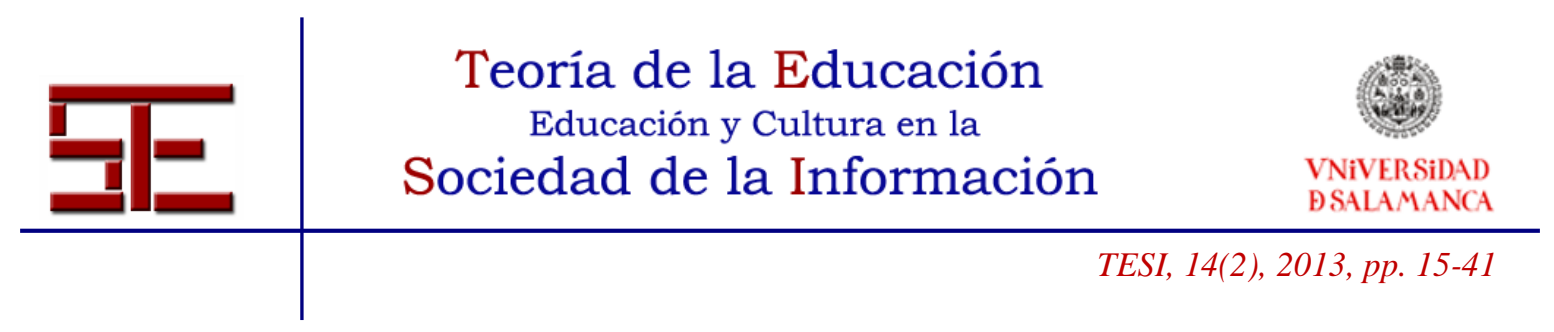

de aprendizaje y TIC (y sus expresiones sinonímicas), comprobaremos que las investigaciones más recientes se agrupan en cinco grandes bloques.

Permanece una línea de investigación más clásica consistente en mostrar que determinados usos de las TIC promueven un determinado tipo de estrategias de aprendizaje que a su vez se asocian con otra $\mathrm{u}$ otras variables psicoeducativas como motivación, metacognición, auto-eficacia, orientación a metas, concepciones y creencias, estilos y enfoques de aprendizaje y un largo etcétera (ver, por ejemplo, Merlo et al., 2007; Valentín et al., 2013). En términos generales, las estrategias de alto nivel, las que suponen un mayor nivel de organización, elaboración y autorregulación, y la motivación intrínseca, relativa al gusto por aprender, se asocian a tareas más abiertas (por ejemplo, las derivadas del trabajo en plataformas) y a la participación de habilidades metacognitivas, mientras que las tareas de ejercitación y las tareas reproductivas se vinculan a estrategias de bajo nivel y a formas de motivación extrínseca (por ejemplo, calificaciones).

Los otros cuatro bloques se refieren a: 1) el uso de las TIC para un mayor ajuste y personalización de los contenidos a aprender a las estrategias de aprendizaje individuales o grupales; 2) la utilización de simuladores y emuladores, y muy especialmente de los juegos, como potenciadores de algunas estrategias de aprendizaje; 3) la aplicación de las TIC para favorecer la auto-, co- y heterorregulación de los procesos instruccionales, y 4) las redes sociales y el concepto de Web 2.0 como marco privilegiado para el desarrollo de estrategias de aprendizaje. A continuación comentamos brevemente cada uno de estos focos de investigación.

1. Personalización de los contenidos a aprender. Una de las conjunciones más productivas entre TIC y estrategias de aprendizaje consiste en la creciente posibilidad de aproximar los contenidos curriculares al perfil de aprendizaje del alumnado y en especial a sus modalidades de aprendizaje, sea para ajustarse a ellas o para optimizarlas. Las investigaciones en este ámbito se centran en sistemas de formación a distancia ( $e$ learning) y combinan, en mayor o menor medida, tutores humanos con tutores artificiales, para obtener información relevante de los destinatarios. Por ejemplo, Wong, Leung, Kwan y Tsang (2010) emplean un sistema de evaluación cognitiva y adaptación a las debilidades y fortalezas de cada alumno mediante un sistema de inteligencia artificial (Web-Based Intelligent Tutoring System), mientras que la propuesta de Poulova y Simonova (2012) se basa en una plataforma flexible que los tutores humanos adaptan a las necesidades, enfoques y estrategias de aprendizaje de cada estudiante.

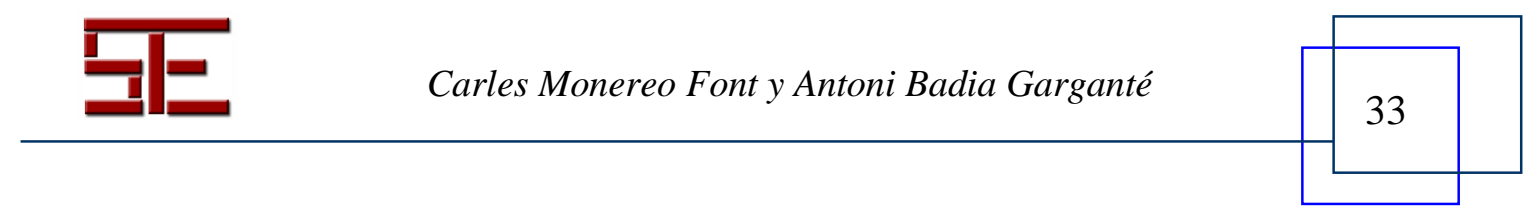




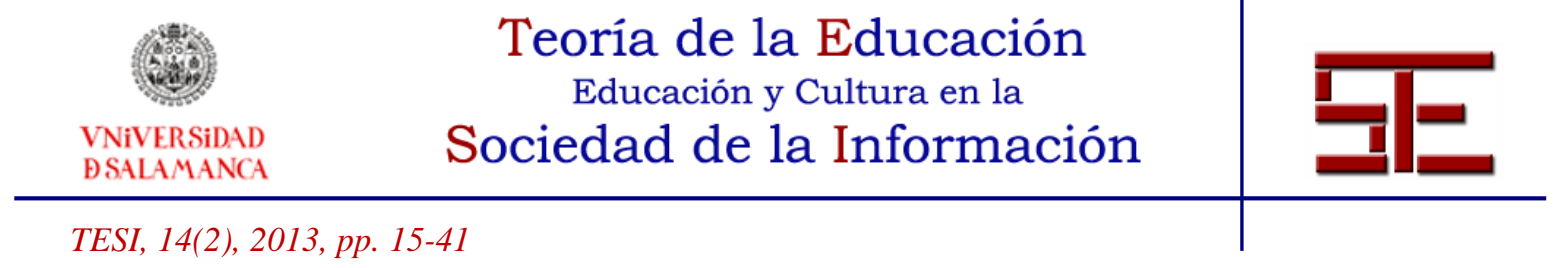

2. Simulación y juegos en los procesos de enseñanza y aprendizaje. El desarrollo de la Inteligencia artificial $\mathrm{y}$, en especial, de escenarios de aprendizaje simulados que gradualmente adoptan formatos de juegos interactivos (se empieza a emplear el barbarismo "gamerización" de las simulaciones) está experimentando un desarrollo espectacular. En el trabajo pionero de Yee (2006) ya se trataba del impacto de los Massive Multiplayer Online Role Playing Games (más conocidos por sus siglas MMORPGs) en el que múltiples participantes (humanos y agentes artificiales) interactúan simultáneamente en escenarios sociales cada vez más complejos y realistas en los que desarrollan distintas identidades y formas de relacionarse y aprender. Los prototipos aplicados a contenidos educativos no han tardado en aparecer y ya existen proyectos como QuizMASter (Multi-Agent Game-Style Learning Activity), en el que un conjunto de "agentes pedagógicos" se encargan de evaluar distintos parámetros del aprendizaje del alumno, entre ellos sus formas de abordar el aprendizaje, dándole un feedback más ajustado para favorecer su motivación y su rendimiento (Dutchuk, Muhammadi y Lin, 2009). Por otra parte, los sistemas de aprendizaje basados en juegos de simulación (Gamelike learning systems) se han mostrado especialmente eficaces con alumnos con bajo nivel de conocimientos y rendimiento académico al proporcionarles estrategias de aprendizaje de diversa índole (lectura del contexto, toma de decisiones, planificación, autorregulación, evaluación, etc.) que les permiten aprovechar al máximo la interacción proporcionada por los juegos (Zhong-Zheng, Cheng y Liu, 2013).

3. Las TIC y la regulación de los procesos instruccionales. Ahora más que nunca las TIC pueden ayudar a efectuar un seguimiento exhaustivo de los progresos de los alumnos o de la marcha de una clase, proporcionando datos detallados de múltiples parámetros, obtenidos mediante las acciones realizadas directamente en el propio ordenador (por ejemplo, a través de plataformas que recogen el número y tipo de intervenciones realizadas o por programas como camtasia, que graban todos los movimientos que efectúa el alumno, o de sistemas basados en eye-tracking que tratan de extrapolar las formas de pensamiento y aprendizaje a través de los movimientos oculares de los usuarios-aprendices, en la pantalla del ordenador), o mediadas por instrumentos como cuestionarios, guías, foros y chats digitales, video-entrevistas, etc. que permiten realizar un análisis posterior del contenido. Por citar dos investigaciones recientes, Levinsen (2011) trata de promover lo que denomina aprendizaje auto-iniciado y auto-programado (self-initiated \& self-programming learning), una suerte de aprendizaje estratégico, a través de una guía de preguntas que pretenden autorregular el aprendizaje de los alumnos (¿Cómo actúo con el fin de explorar información? ¿Cuándo prefiero colaborar?, ¿Qué

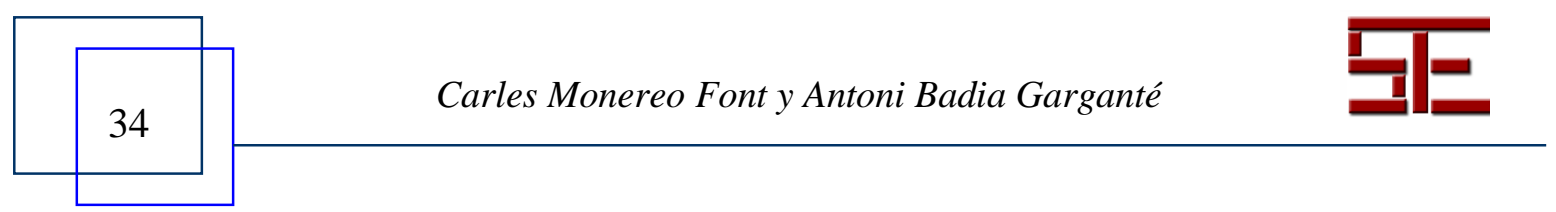




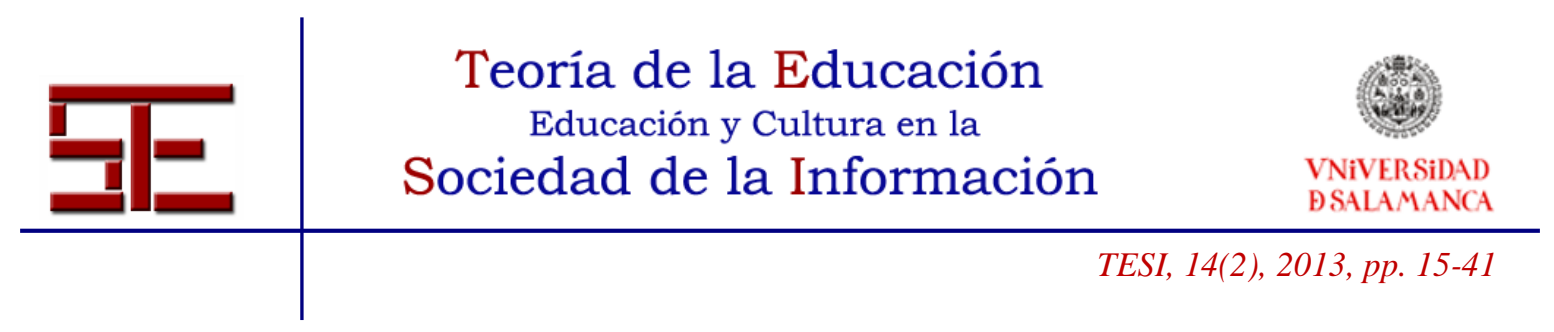

obstáculos o retos suelo encontrar y cómo actúo frente a ellos? ¿Qué tipo de ayudas me permiten eludir esos obstáculos o resolver problemas? ¿Cómo puedo cambiar mi estrategia?, etc.). El investigador hace que esta actividad sea visible para el grupo y establece un sistema de ayuda recíproca entre los participantes para que se ofrezcan "andamiajes" unos a otros.

Una aproximación distinta consiste en proporcionar a los estudiantes y/o profesores información relevante sobre la dinámica del aula y los procesos de enseñanza-aprendizaje para que ambos colectivos reflexionen sobre sus prácticas y adopten estrategias más ventajosas para enseñar y aprender. Por ejemplo, en el proyecto SharEvents (Monereo, Panadero y Scardezini, 2013) docentes y estudiantes responden semanalmente a un cuestionario on-line, de forma independiente (y anónima en el caso de los estudiantes) sobre el funcionamiento de la clase, la marcha de los equipos de trabajo, sobre sus dudas y progresos, sobre los incidentes acaecidos y su adecuada resolución, etc., y tras unos meses, reciben la información resumida de los otros participantes (el profesor de sus alumnos y los alumnos de sus compañeros y del profesor). Ello les obliga a replantearse sus estrategias y sus formas de abordar los problemas y conflictos surgidos durante la dinámica de clase.

4. Redes sociales y potenciación de estrategias de aprendizaje. Aunque tradicionalmente las redes sociales y la Web 2.0 se asocian a ideas como interaccionar, compartir y colaborar activamente, su utilización educativa puede ser extremadamente conservadora, centrada en el docente y de un impacto limitado en los aprendizajes (Tambouris et al., 2012). Sin embargo, se trata del área de investigación que actualmente presenta un mayor dinamismo debido, entre otras razones, a la enorme extensión de su uso, casi a nivel planetario y la facilidad de recopilar y analizar datos, sin el coste que supone la creación de artefactos y sistemas especialmente diseñados. En estos momentos se están efectuando investigaciones relevantes en relación al impacto de las principales redes sociales, como Facebook (Mack, Behler, Roberts y Rimland, 2007; Shiu, Fong y Lam, 2010) o Twitter (Lin, Hoffman y Borengasser, 2013), sobre las prácticas de aprendizaje de los estudiantes.

Quizás el concepto que mejor agrupe este conjunto de innovaciones e investigaciones sea el de aprendizaje móvil (Mobile Learning o m-learning, en contraposición con el $e$ learning) que defiende una enseñanza basada en el uso de todo tipo de dispositivos móviles (smartphones, tabletas digitales, agendas electrónicas o PDA, etc.) en los que puedan integrarse toda suerte de experiencias educativas a través de redes sociales, miniconferencias, wikis, lecturas escogidas, visitas guiadas, etc. Un buen ejemplo de esa

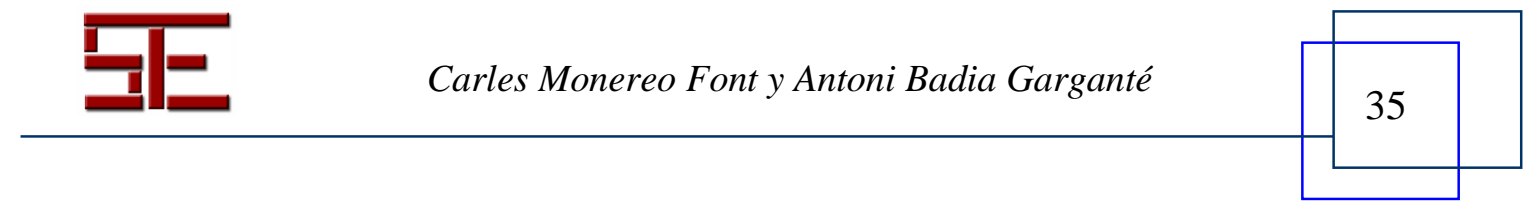




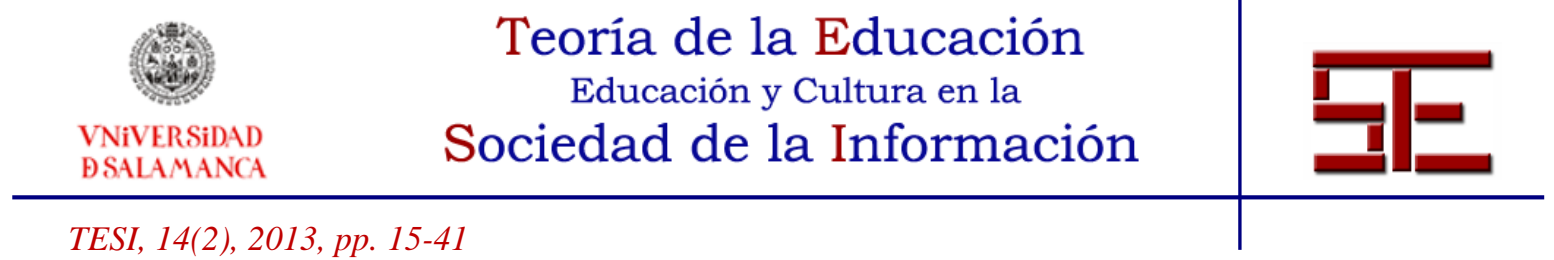

integración es la investigación de Menkhoff y Bengtsson (2012), que parte de un aprendizaje semi-presencial (Blended Learning o b-learning), en la que se combinan las clases habituales con un sistema de m-learning en el que los estudiantes realizan trabajos de campo, lecturas, visitas académicas, guiados en todo momento por sus profesores y otros compañeros, con los que se hallan continuamente conectados.

Una de las características más evidentes de todos estos desarrollos es que, en primer lugar, no se trata de líneas de investigación aisladas, sino que se entrecruzan, influyen y retroalimentan unas a otras, permanentemente. De este modo, algunos dispositivos inteligentes pueden aplicarse a algunas redes sociales para lograr que la participación de sus usuarios sea más autorregulada y relevante para toda la comunidad, produciendo una especie de "conocimiento colectivo" enormemente valioso (un ejemplo paradigmático sería el Facebook: http://www.patientslikeme.com/, en el que los participantes-pacientes aprenden de las experiencias de otros).

El segundo denominador común de estos enfoques de la investigación es una tendencia a lo que podríamos denominar una enseñanza y aprendizaje "ecológicos" en el sentido de que se buscan sistemas para rentabilizar las actividades cotidianas que realizan alumnos y profesores de manera que, sin disponer de estructuras de formación intencionales, éstos adquieran conocimientos, estrategias y competencias discentes y docentes. Dicho de otro modo, la formación dominante ad hoc resulta cara, insuficientemente contextualizada y sus efectos son difíciles de transferir y permanecen poco en el tiempo. Utilizar las redes sociales, los juegos, los cuestionarios one-line, las plataformas de aprendizaje, los teléfonos móviles, etc., para, por ejemplo, promover nuevas estrategias de aprendizaje y enseñanza, resulta menos costoso, menos invasivo, más motivante y probablemente más sostenible en el tiempo.

Como dijo Marx (Groucho), el futuro ya no es lo que era, y lo único que podemos decir, a ciencia cierta, es que aunque las formas de enseñanza y aprendizaje que deberán afrontar nuestros hijos y nietos requieran nuevas estrategias, la competencia para aprender a aprender será cada vez más cuestión de supervivencia.

\section{6.- BIBLIOGRAFÍA}

Azevedo, R. y Hadwin, A. F. (2005). Scaffolding self-regulated learning and metacognition. Implications for the design of computer-based scaffolds. Instructional Science, 33 (5), 367-379.

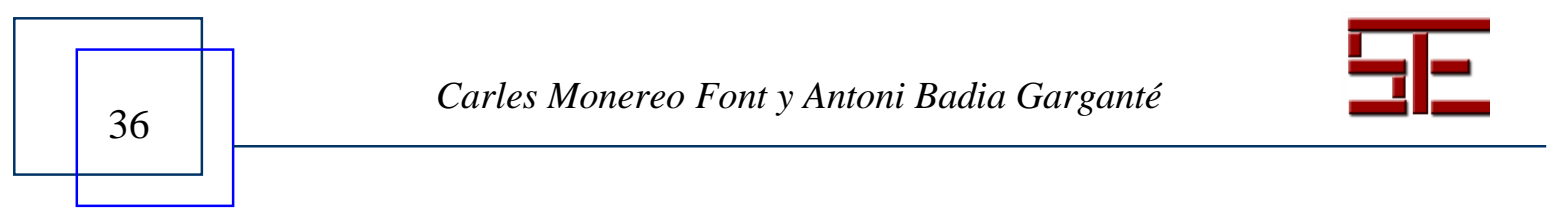




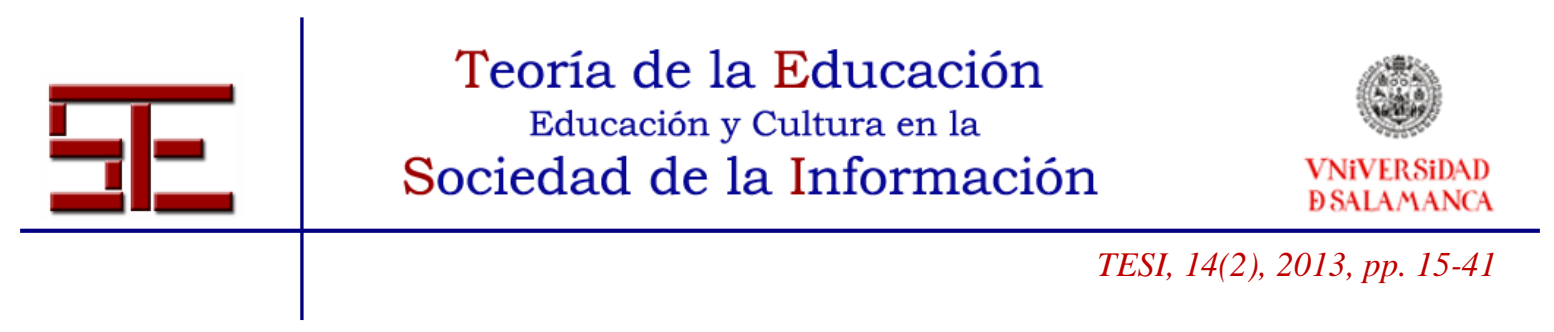

Azevedo, R., Johnson, A., Chauncey, A. y Graesser, A. (2011). Use of hypermedia to assess and convey self-regulated learning. En B. J. Zimmerman y D. H. Schunk (Eds.), Handbook of self-regulation of learning and performance (pp. 102-121). New York: Taylor \& Francis.

Badia, A. (Coord.), Álvarez, I., Carretero, R., Liesa, E. y Becerril, L. (2012). Estrategias y competencias de aprendizaje en educación. Madrid: Editorial Síntesis.

Badia, A., Becerril, L. y Romero, M. (2010). La construcción colaborativa de conocimiento en las redes de comunicación asíncrona y escrita (RCAE): una revisión de los instrumentos analíticos. Cultura y Educación, 22 (4), 455-474.

Badia, A. y Monereo, C. (2008). La enseñanza y el aprendizaje de estrategias de aprendizaje en entornos virtuales. En C. Coll, y C. Monereo (Eds.), Psicología de la educación virtual (pp. 348-367). Madrid: Morata.

Barberà, E. y Badia, A. (2005). Hacia el aula virtual: actividades de enseñanza y aprendizaje en la red. Revista Iberoamericana de Educación, 36 (9), 1-21.

Becerril, L. y Badia, A. (en prensa). La competencia informacional en la Educación Secundaria. Demanda de aprendizaje y resolución colaborativa de problemas informacionales con apoyo de las TIC. Revista de Educación.

Coll, C., Mauri, M. y Onrubia, J. (2008). La utilización de las tecnologías de la información y la comunicación en la educación. Del diseño tecno-pedagógico a las prácticas de uso. En C. Coll y C. Monereo (Eds.), Psicología de la educación virtual (pp. 74-104). Madrid: Morata.

Du Plessis, A. y Webb, P. (2011). An extended Cyberhunts strategy: Learner centred learning-by-design. Australian Journal of Educational Technology, 27 (7), 1190-1207.

Dutchuk, M., Muhammadi, K. A. y Lin, F. (2009). QuizMASter - A multi-agent gamestyle learning activity. En Proceedings of the 4th International Conference on ELearning and Games (pp. 263-272). Banff, Canada: Learning by Doing.

Fischer, F., Kollar, I., Stegmann, K. y Wecker, C. (2013). Toward a script theory of guidance in Computer-Supported Collaborative Learning. Educational Psychologist, 48 (1), 56-66.

Gargallo, B. (2012). Un aprendiz estratégico para una nueva sociedad. Revista Teoría de la Educación: Educación y Cultura en la Sociedad de la Información, 13 (2), 246-272 [Fecha de consulta: 25/04/2013].

http://campus.usal.es/ revistas_trabajo/index.php/revistatesi/article/view/9008/9252.

Gulikers, J., Bastiaens, T. y Kirschner, P. (2004). A five-dimensional framework for Authentic Assessment. Educational Technology Research and Development, 52, 67-86.

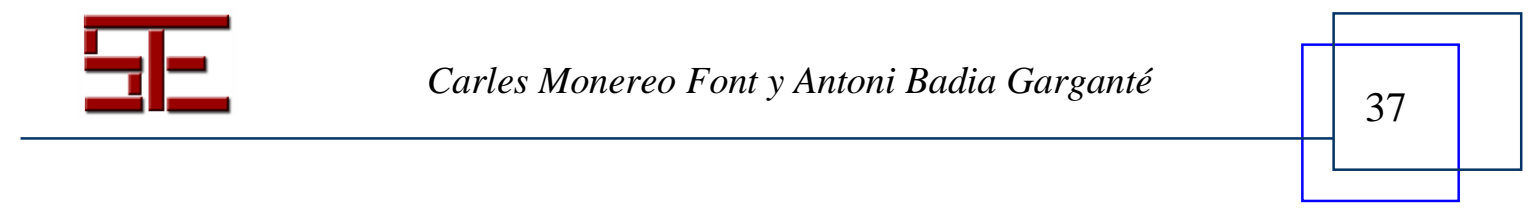




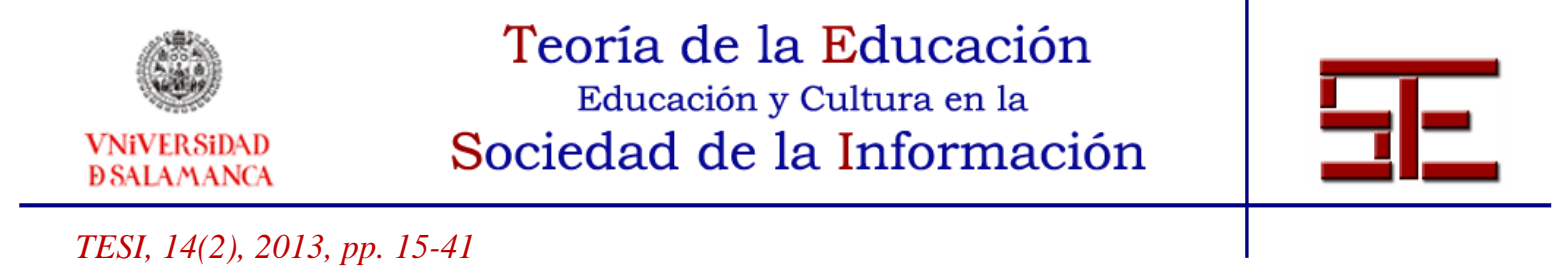

Hadwin, A. F., Järvelä, S. y Miller, M. (2011). Self-regulated, co-regulated, and socially shared regulation of learning. En B. J. Zimmerman y D. H. Schunk (Eds.), Handbook of self-regulation of learning and performance (pp. 65-84). New York: Taylor y Francis.

Herrington, J. y Kervin, L. (2007). Authentic learning supported by technology: 10 suggestions and cases of integration in classrooms. Educational Media International, 44 (3), 219-236.

Hirata, Y. y Hirata, Y. (2010) Students' Attitudes towards Web Searching. En 3rd International Conference on Hybrid Learning. Lecture Notes in Computer Science, 6248, 127-136.

Huang, C. J., Wang, Y. W., Huang, T. H., Chen, Y. C., Chen, H. M. y Chang, S. C. (2011). Performance evaluation of an online argumentation learning assistance agent. Computers \& Education, 57 (1), 1270-1280.

Inan, F., Lowther, D. Ross., S. y Strahl, J. (2010). Pattern of classroom activities during students' use of computers: Relations between instructional strategies and computer applications. Teaching and Teacher Education, 26 (3), 540-546.

Janssen, J., y Bodemer, D. (2013). Coordinated computer-supported collaborative learning: Awareness and awareness tools. Educational Psychologist, 48 (1), 40-55.

Janssen, J., Erkens, G. y Kirschner, P. A. (2011). Group awareness tools: It's what you do with it that matters. Computers in Human Behavior, 27 (3), 1046-1058.

Jonassen, D. H. (2006). Modeling with technology: Mindtools for conceptual change. Columbus OH: Pearson-Prentice Hall.

Lajoie, S. P. y Derry, S. J. (1993). Computers as cognitive tools (vol. 1). Hillsdale, NJ: Lawrence Erlbaum Associates.

Levinsen, K.T. (2011). Fluidity in the Networked Society - Self-initiated learning as a Digital Literacy Competence. Electronic Journal of e-Learning, 9 (1), 52-62.

Lim, C. y Tay, L. (2003). Information and communication technologies (ICT) in an elementary school: Students' engagement in higher order thinking. Journal of Educational Multimedia and Hypermedia, 12 (4), 425-451.

Lin, M., Hoffman, E. y Borengasser, C. (2013). Is Social Media Too Social for Class? A Case Study of Twitter Use. Techtrends: Linking Research \& Practice To Improve Learning, 57 (2), 39-45.

Ljungdahl, L. y Prescott, A. (2009). Teachers' use of diagnostic testing to enhance students' literacy and numeracy learning. International Journal of Learning, 16, 461-476.

Looney, J. (2010). Making it Happen: formative assessment and educational technologies. Promethean Thinking Deeper Research Papers, number 1, part 3.

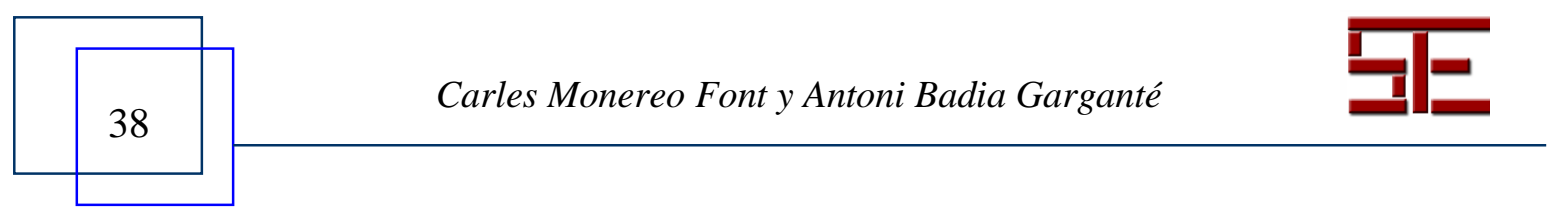




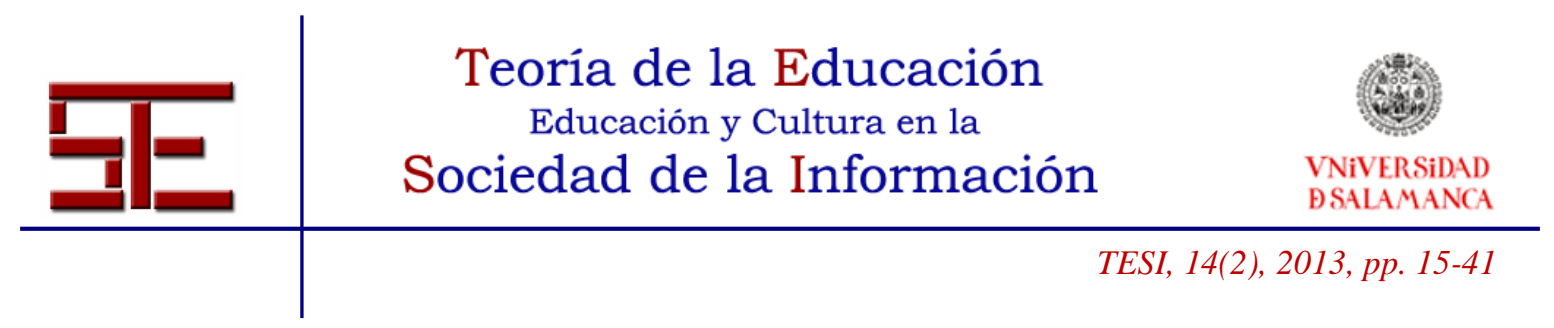

Mack, D., Behler, A., Roberts, B., y Rimland, E. (2007) Reaching Students with Facebook: Data and Best Practices. Electronic Journal of Academic and Special Librarianship, 8 (2). Extraído el: 03/05/2013, en: http://southernlibrarianship.icaap.org/content/v08n02/mack_d01.html.

Menkhoff, T. y Bengtsson, M. (2012). Engaging students in higher education through mobile learning: lessons learnt in a Chinese entrepreneurship course. Educational Research For Policy \& Practice, 11 (3), 225-242.

Merlo, G., Seta, L., Ottaviano, S., Chifari, A., Chaizzese, G., Allegra, M. y Todaro, G. (2007). Guiding students to acquire strategies for Web learning through Gym2Learn. En T. Bastiaens \& S. Carliner (Eds.), Proceedings of World Conference on E-Learning in Corporate, Government, Healthcare, and Higher Education (pp. 7260-7266). Chesapeake, VA: AACE.

Monereo, C. (Coord.) (2005). Internet y competencias básicas. Barcelona: Graó.

- (2009). Aprender a encontrar y seleccionar información: de Google a la toma de apuntes (pp. 89-105) En J. I. Pozo. y M. P. Pérez Echevarría. Psicología del aprendizaje universitario. La formación en competencias. Madrid: Morata.

Monereo, C. y Badia, A. (2012). La competencia informacional desde una perspectiva psicoeducativa: enseñanza en base a problemas prototípicos y emergentes. Revista Española de Documentación Científica, 35 (3), 76-100.

Monereo, C., Castello, M., Clariana, M., Palma, M. y Pérez, M. L. (1994). Estrategias de enseñanza y aprendizaje. Barcelona: Graó.

Monereo, C. y Fuentes, M. (2008). Cómo buscan información en Internet los adolescentes. Investigación en la escuela, 64, 45-58.

Monereo, C., Panadero, E. y Scardezini, R. (2013). SharEVents. La utilización de informes compartidos sobre incidentes críticos como medio para la formación docente. Cadernos de Educação, 42.

Monereo, C. y Pozo, J. I. (2001). Tema del mes: Competencias para sobrevivir en el siglo XXI. Cuadernos de Pedagogía, 298.

Monereo, C. y Romero, M. (2008). Los entornos virtuales de aprendizaje basados en sistemas de emulación socio-cognitiva. En C. Coll, y C. Monereo (Eds.), Psicología de la educación virtual (pp. 194-212). Madrid: Morata.

Neo, M., Neo, K. y Tan, H. (2012). Applying authentic learning strategies in multimedia and Web Learning Environment (MWLE): Malaysian students' perspective. Turkish Online Journal of Educational Technology, 11 (3), 50-60.

Nunan, D. (2010). Technology Supports for Second Language Learning. International Encyclopedia of Education, 8, 204-209.

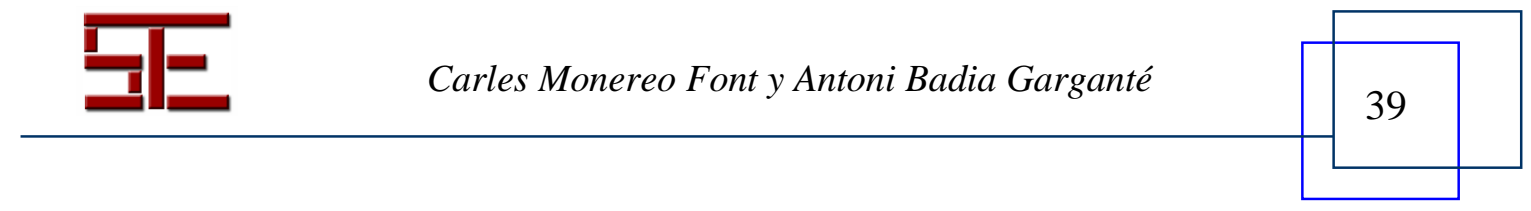




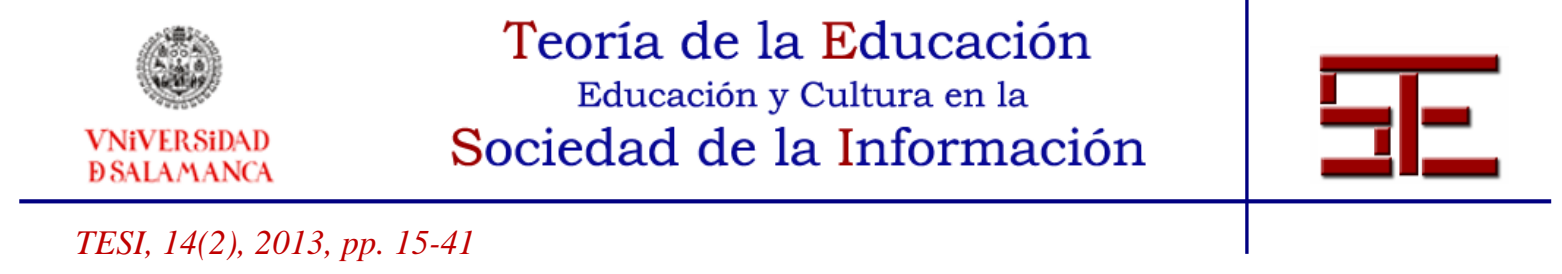

Pea, R. D. (1985). Beyond amplification: Using the computer to reorganize mental functioning. Educational Psychologist, 20 (4), 167-182.

Poulova, P. y Simonova, I. (2012). Flexible E-learning: Online Courses Tailored to Student's Needs. En 9th International Scientific Conference on Distance Learning in Applied Informatics; Conference proceedings; (pp.251-260).

Prensky, M. (2011). Enseñar a nativos digitales. Madrid: Ediciones SM.

Raes, A., Schellens, T., De Wever, B. y Vanderhoven, E. (2012). Scaffolding information problem solving in web-based collaborative inquiry learning. Computers \& Education, 59 (1), 82-94.

Redecker, C. y Johannessen, Ø. (2013). Changing Assessment-Towards a New Assessment Paradigm Using ICT. European Journal of Education, 48 (1), 79-96.

Reusser, K. (1993). Tutoring systems and pedagogical theory: Representational tools for understanding, planning and reflection in problem solving. En S. Lajoie y S. J. Derry (Eds.), Computers as cognitive tools (pp. 143-177). Hillsdale, NJ: Lawrence Erlbaum Associates.

Salomon, G. (1985). Information technologies: What you see is not (always) what you get. Educational Psychologist, 20 (4), 207-216.

- (1992). Las diversas influencias de la tecnología en desarrollo de la mente. Infancia y Aprendizaje, 58, 143-159.

Salomon, G., Perkins, D. N. y Globerson, T. (1992). Coparticipando en el conocimiento: la ampliación de la inteligencia humana con las tecnologías inteligentes. Comunicación, Lenguaje y Educación, 13, 6-22.

Scherer, R. y Tiemann, R. (2012). Factors of problem-solving competency in a virtual chemistry environment: The role of metacognitive knowledge about strategies. Computers \& Education, 59 (4), 1199-1214.

Selwyn, N. y Bullon, K. (2000). Primary school children's use of ICT. British Journal of Educational Technology, 31 (4), 321-332.

Shiu, H., Fong, J. y Lam, J. (2010). Facebook - Education with Social Networking Websites for Teaching and Learning. International Conference on Hybrid Learning, Conference proceedings, (pp.59-70).

Stein, S.J., Isaacs, G. y Andrews, T. (2004). Incorporating Authentic Learning Experiences Within a University Course. Studies in Higher Education, 29, 239-258.

Tambouris, E., Panopoulou, E., Tarabanis, K., Ryberg, T., Buus, L., Peristeras, V., Lee, D. y Porwol, L. (2012). Enabling Problem Based Learning through Web 2.0 Technologies: PBL 2.0. Educational Technology \& Society, 15 (4), 238-251.

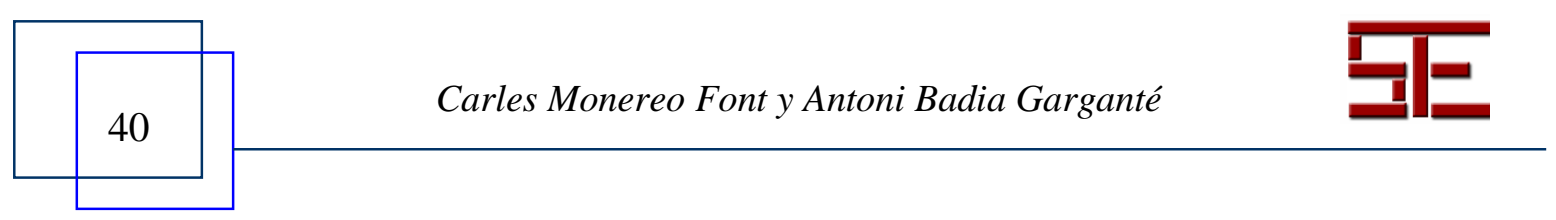




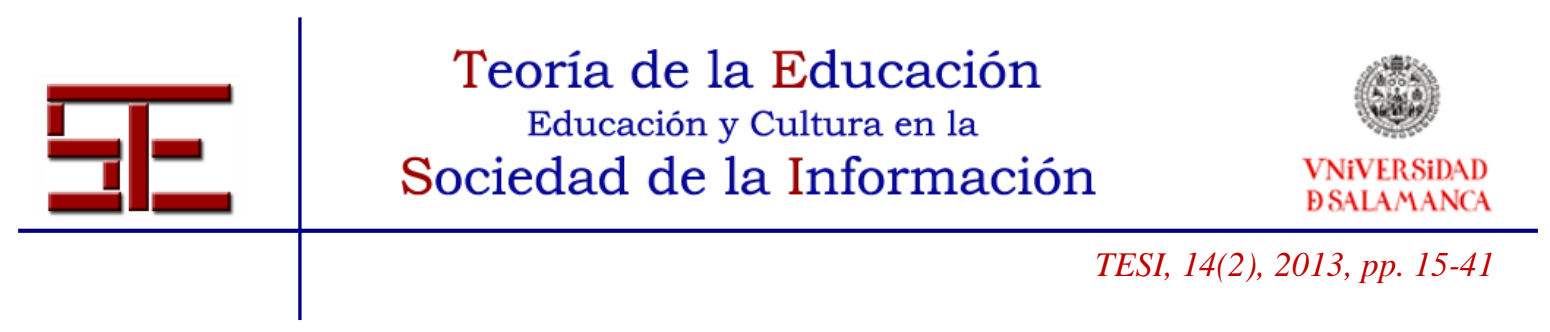

Tiana, A. (2011). Análisis de las competencias básicas como núcleo curricular en la educación obligatoria española. Bordón, 63 (1), 63-75.

Valentín, A., Mateos, P., González-Tablas, M., Pérez, L., López, E. y García, I. (2013) Motivation and learning strategies in the use of ICTs among university students. Computers \& Education, 61, 52-58.

Van den Boom, G., Paas, F. y Van Merriënboer, J. J. (2007). Effects of elicited reflections combined with tutor or peer feedback on self-regulated learning and learning outcomes. Learning and Instruction, 17 (5), 532-548.

Van den Boom, G., Paas, F., Van Merriënboer, J. J. y Van Gog, T. (2004). Reflection prompts and tutor feedback in a web-based learning environment: Effects on students' self-regulated learning competence. Computers in Human Behavior, 20 (4), 551-567.

Weinberger, A., Ertl, B., Fischer, F. y Mandl, H. (2005). Epistemic and social scripts in computer-supported collaborative learning. Instructional Science, 33 (1), 1-30.

Wong, K., Leung, K., Kwan, R. \& Tsang, P. (2010). E-Learning: Developing a Simple Web-Based Intelligent. Tutoring System Using Cognitive Diagnostic Assessment and Adaptive Testing Technology. Hybrid Learning, 6248, 23-34.

Yee, N. (2006). The Psychology of MMORPGs: Emotional Investment, Motivations, Relationship Formation, and Problematic Usage. En R. Schroeder \& A. Axelsson (Eds.), Avatars at Work and Play: Collaboration and Interaction in Shared Virtual Environments (pp. 187-207). London: Springer-Verlag.

Zhong-Zheng, L., Cheng, Y. y Liu, Ch. (2013). A constructionism framework for designing game-like learning systems: Its effect on different learners. Bristish Journal of Educational Technology, 44 (2), 208-224.

Zimmerman, B. J. y Schunk, D. H. (Eds.) (2011). Handbook of self-regulation of learning and performance. New York: Taylor y Francis.

Para citar el presente artículo puede utilizar la siguiente referencia:

Monerero Font, C. y Badia Garganté, A. (2013). Aprendizaje estratégico y tecnologías de la información y la comunicación: una revisión crítica. Revista Teoría de la Educación: Educación y Cultura en la Sociedad de la Información. 14(2), 15-41 [Fecha de consulta: $\mathrm{dd} / \mathrm{mm} / \mathrm{aaaa}]$.

http://campus.usal.es/ revistas_trabajo/index.php/revistatesi/article/view/10212/10622

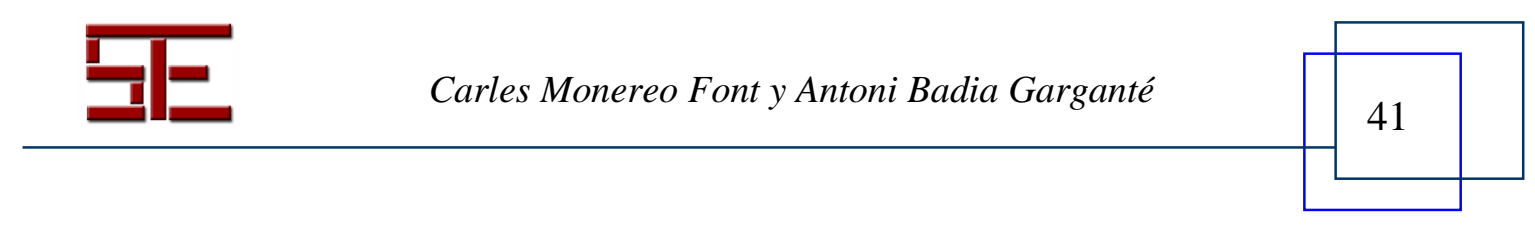

